\title{
Messung der Zerfallszeiten von Molekülionen
}

\author{
Ch. Ottinger
}

Physikalisches Institut der Universität Freiburg i. Br.

(Z. Naturforschg. 22 a, 20-40 [1967] ; eingegangen am 3. Oktober 1966)

\begin{abstract}
The time interval between the ionization of a molecule by electron impact and its dissociation was determined from the kinetic energy of the fragment ions. This technique allows a direct differential sampling of the dissociation rate at various times, in contrast to previous, integral methods. The use of a disc-shaped molecular beam of $28 \mu$ half-width and a draw-out field of $10000 \mathrm{~V} / \mathrm{cm}$ in the ionization region resulted in a minimum measured time interval of $7.4 \cdot 10^{-9} \mathrm{sec}$. The longest times measured were between 5 and $6 \mu$ secs. The time dependence of the decomposition rate was measured for two decompositions in butane, five in heptane, three in benzene, one in $\mathrm{CD}_{4}$ and one in $\mathrm{CO}_{2}^{++}$, with rather similar results. It was established that the decay is not exponential. All decay curves, plotted semi-logarithmically, were found to have an increasing slope towards shorter times, indicating contributions from a variety of rate constants, in agreement with the predictions of the statistical theory of ion decompositions. 50 delayed decompositions in the $\mu$ sec region were investigated, about half of which had not been reported previously. For these measurements the detection limit was about $10^{-5}$ of the base peak intensity. In particular, such "metastables" were found in methane and the deutero-methanes. They were explained by con. sidering the external rotation of the ion. Small fragmentation energies set free during the decomposition can be determined from the kinetic ion energy. The detection limit is as low as $5 \mathrm{mV}$ for the total fragmentation energy, since the method is unaffected by thermal translational energy. Some rules concerning these energies were found.
\end{abstract}

Eines der wichtigsten Probleme der Reaktionskinetik ist die Berechnung von Reaktionskonstanten unimolekularer Zerfälle. Die ursprünglich für chemische Reaktionen entwickelten Ideen wurden von Rosensтоск u. a. ${ }^{1}$ auf Ionen übertragen. Er begründete damit die statistische Theorie der Ionenzerfälle, die in der Folge häufig diskutiert und angewendet wurde. Die ersten Messungen von Zerfallskonstanten bei Ionen stammen von Hipple ${ }^{2}$, der die sogenannten metastabilen Molekülionen mit Zerfallszeiten von der Größenordnung einiger $\mu$ sec untersuchte. Seitdem sind verschiedene Versuche unternommen worden, den beobachtbaren Zeitbereich solcher Zerfälle nach längeren ${ }^{3-6}$ und kürzeren ${ }^{7-10}$ Zeiten hin zu erweitern. Davon hatten besonders die letzteren Arbeiten sich widersprechende Ergebnisse. Das Ziel der vorliegenden Arbeit war, die Bildungshäufigkeit der Fragmentionen über einen möglichst weiten Bereich von Zeiten zwischen Ionisation und Dissoziation zu messen, anfangend bei möglichst kurzen Zeiten. Diese experimentelle Information über die Zerfallskonstanten $k$ sollte eine besonders direkte Prüfung der statistischen Theorie ermöglichen.

1 H. M. Rosenstock, M. B. Wallenstein, A. L. Wahrhaftig u. H. Eyring, Proc. Natl. Acad. Sci. U.S. 38, 667 [1952].

2 J. A. Hipple, Phys. Rev. 71, 594 [1947].

3 M. E. Wacks u. A. L. Wahrhaftig, Univ. of Utah, Techn. Report VII, 1958.

4 U. v. Zahn u. H. Tatarcyk, Phys. Lett. 12, 190 [1964].

5 H. Tatarcyk u. U. v. Zahn, Z. Naturforschg. 20 a, 1708 [1965].

\section{Prinzip der Messung}

Wenn ein Molekülion während seiner Beschleunigung in einem elektrischen Feld ein neutrales Fragment abspaltet, so hängt die kinetische Energie des Restions vom Zeitpunkt dieser Dissoziation ab. Dies kommt daher, daß bei einer Dissoziation im Fluge die kinetische Energie des dissoziierenden Ions $\mathrm{AB}^{+}$ sich im Verhältnis der Massen auf die Bruchstücke $\mathrm{A}^{+}$und $\mathrm{B}$ verteilt:

$$
\begin{array}{ll}
\text { Prozeß: } & \mathrm{AB}^{+} \rightarrow \mathrm{A}^{+}+\mathrm{B}, \\
\text { Massen: } & m_{0}=m+\Delta m, \\
\text { kin. Energien: } & m_{0} v^{2}=\frac{m v^{2}}{2}+\frac{\Delta m v^{2}}{2}
\end{array}
$$

(Hierbei sind etwaige bei der Dissoziation freiwerdende Anfangsenergien vernachlässigt.) Das hier verwendete Verfahren zur Messung der Zeit zwischen Ionisation und Dissoziation eines Moleküls beruht daher auf einer Messung der kinetischen Energie der Ionen.

In dem Experiment wird zuerst dafür gesorgt, daß alle Ionen auf dem gleichen Potential in dem

${ }^{6}$ F. Meter u. A. G. Harrison, J. Chem. Phys. 43, 1778 [1965].

7 J. C. Schug, J. Chem. Phys. 40, 1283 [1964].

8 N. D. Coggeshall, J. Chem. Phys. 37, 2167 [1962].

9 J. Momigny, Bull. Soc. Chim. Belg. 70, 291 [1961].

10 G. V. Karachevtsev u. V. L. Talrose, Kinetika i Katalis IV, 923 [1963]. 
homogenen Ionenbeschleunigungsfeld starten, indem das zu untersuchende Gas in einem sehr dünnen Molekularstrahl längs einer Äquipotentialfläche eingeschossen wird. Die Elektronen werden durch Löcher in der positiven Feldelektrode (Ionenrepeller) senkrecht in den Molekularstrahl geschossen. Sie durchsetzen ihn zweimal, da sie ihre Bewegung im Ionenziehfeld umkehren. Hat die Ebene des Molekularstrahls das Potential $U$, so durchlaufen die dort gebildeten Ionen $\mathrm{AB}^{+}$die auf Erdpotential liegende Apparatur mit der kinetischen Energie $e U$, falls sie nicht dissoziieren. Dasselbe gilt für Bruchstückionen $\mathrm{A}^{+}$, die aus $\mathrm{AB}^{+}$praktisch augenblicklich, d. h. im Moment der Ionisation, entstehen. Erfolgt dagegen die Bildung von $\mathrm{A}^{+}$mit einer Zeitverzögerung $t$ gegenüber der Ionisation, so hat das Mutterion $\mathrm{AB}^{+}$ in der Zeit $t$ im Ionenziehfeld $E$ die kinetische Energie $(e|\mathbb{E}| t)^{2} / 2 m_{0}$ aufgenommen, von der bei der Dissoziation der Bruchteil $\Delta m / m_{0}$ auf das neutrale Bruchstück entfällt. Das geladene Bruchstück $\mathrm{A}^{+}$hat daher nach Durchlaufen der gesamten Beschleunigungsspannung eine um

$$
e . \Delta U=\frac{\Delta m}{m_{0}} \cdot \frac{1}{2 m_{0}}(e|\tilde{E}| t)^{2}
$$

kleinere kinetische Energie als $e U$, die Energie der Mutterionen (es scheint von einem Entstehungsort zu kommen, dessen Potential um $\Delta U$ unterhalb des Strahlpotentials liegt). Durch Messung dieses Energieverlustes $e \Delta U$ läßt sich die gesuchte Zeit $t$ bestimmen.

Die Messung der kinetischen Energie der Ionen geschieht in einem Zylinderkondensator. Nach Passieren des Kondensators werden die Ionen in einem magnetischen Sektorfeld nach Massen getrennt und nachgewiesen. Um bei der Messung einer bestimmten Fragmentmasse $m^{+}$den Magnetstrom konstant lassen zu können, wird zur Energiemessung auch die Spannung am Zylinderkondensator konstant gelassen und statt dessen das Ionenquellenpotential variiert. Der Durchlaßbereich des Kondensators hat dann immer die gleiche Breite auf der Energieskala. Damit diese möglichst klein wird, müssen die Ionen den Kondensator relativ langsam (z. B. mit $500 \mathrm{eV}$ ) durchlaufen. Andererseits ist zur Messung kurzer Zeiten nach (1) eine hohe Feldstärke nötig, die bei einem bequem einzustellenden Abstand zwischen Ionenrepeller und -Ziehblende auch eine hohe Ionenbeschleunigungsspannung erforderlich macht. Die Ionen müssen also vor ihrem Eintritt in den Kondensator abgebremst werden.
Abb. 1 zeigt den so erhaltenen Potentialverlauf schematisch. Die gestrichelte Linie gibt ein variiertes Ionenquellenpotential an.

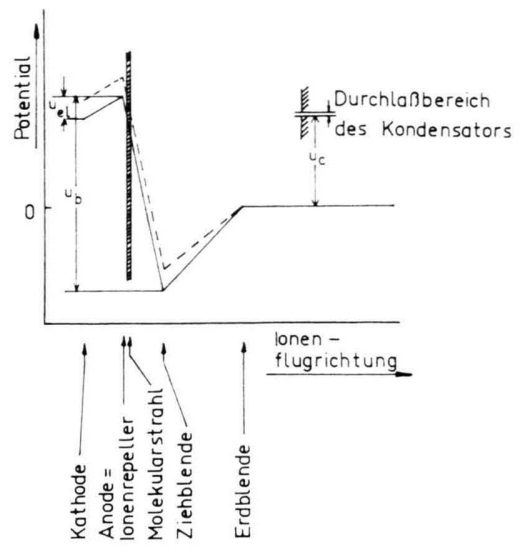

Abb. 1. Potentialverlauf in der Ionenquelle.

\section{Beschreibung der Apparatur}

Um Zeiten von ca. $10^{-8}$ sec meßbar zu machen, braucht man einen Molekularstrahl, dessen Breite in der Größenordnung 10-100 $\mu$ liegt. Zur Erzielung einer ausreichenden Intensität wurden die den Molekularstrahl kollimierenden beiden Spalte als konzentrische Ringspalte ausgebildet.

Abb. 2 zeigt die Ionenquelle und Ionenoptik halbschematisch; die wesentlichen Teile sind maßstäblich gezeichnet. Die Zeichenebene liegt in Wirklichkeit horizontal.

Die linken Spaltbacken der Spalte $S_{1}$ und $S_{2}$ sind die Stirnflächen von zwei $3 \mathrm{~mm}$ breiten zylindrischen Ringen, die aus dem massiven zylindrischen Block A herausragen. Sie liegen in einer Ebene und sind bis auf wenige $\mu$ plan geschliffen. Die rechten Spaltbacken werden durch die Platte $\mathrm{P}$ gebildet, deren linke Fläche ebenfalls geschliffen ist. Die Breite der Spalte wurde durch Streifchen aus Kupferfolie, die zwischen P und A geklemmt wurden, auf $20 \mu$ eingestelt, bei einigen Versuchen auf nur $5 \mu$. Das Gas wurde in den Raum zwischen A und dem Metallbalg B durch das Röhrchen R eingelassen. Bei $\mathrm{G}$ ist der Balg gegen $\mathrm{P}$ mit Golddraht abgedichtet. Das durch $\mathrm{S}_{1}$ mit einer breiten Winkelverteilung ausströmende Gas bildet mit dem kleinen Teil, der durch $\mathrm{S}_{2}$ gelangt, den gewünschten Strahl, der größte Teil wird aber nach links hin durch eine $250 \mathrm{l}$. Öldiffusionspumpe und gegebenenfalls durch eine Kühlfalle abgepumpt. Im Experimentiergebiet im Zentrum der Ringspalte wird so eine "Gasscheibe“ von geringer Dicke und relativ hoher Dichte erzeugt.

Die Kathode K in dem Kathodentöpfchen KT besteht aus zwei Wolframbändchen von $2 \mathrm{~mm}$ Breite im Abstand von einigen mm von der Anode AN, die das Kathodentöpfchen abschließt und gleichzeitig als Ionenrepeller fungiert. Sie besteht aus einer $0,1 \mathrm{~mm}$ dicken 


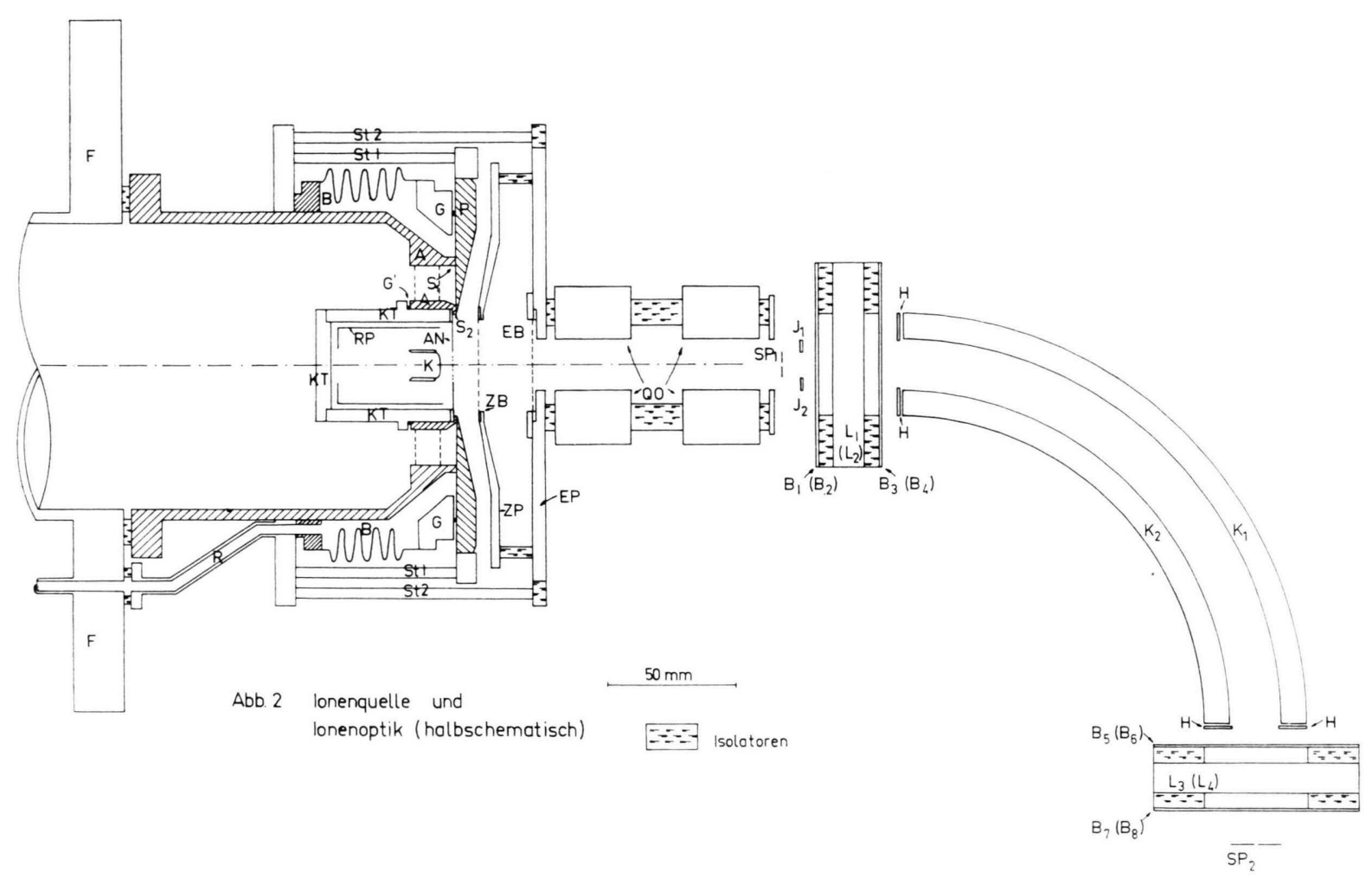

Abb. 2. Ionenquelle und Ionenoptik (halbschematisch) .

Kupferfolie, die in ihrem mittleren Bereich durchlöchert ist. Die gemessene Transmission für Elektronen war bei verschiedenen Elektronenbeschleunigungsspannungen und Elektronenströmen immer etwa 10\%, die Gesamtemission betrug meist ca. $1 \mathrm{~mA}$. RP ist ein zylindrischer Elektronenrepeller. Im Betrieb erreichte A ca. $150{ }^{\circ} \mathrm{C}$, die Gastemperatur dürfte dann bei allen Experimenten ebenfalls $150{ }^{\circ} \mathrm{C}$ oder etwas weniger betragen haben.

Der ganze Block A liegt auf Anodenpotential. EP ist eine geerdete Platte mit der „Erdblende“ EB. Auf EP ist die Platte ZP mit der „Ziehblende“ ZB hochspannungsisoliert aufgebaut. $\mathrm{ZB}$ und $\mathrm{EB}$ tragen sehr gut ebene Netze aus Kupfer (34 $\mu$ Gitterkonstante, ca. 75\% Transmission). Die Abstände AN - ZB (Ionenziehfeld) und $\mathrm{ZB}-\mathrm{EB}$ (Ionenbremsfeld) betragen $10 \mathrm{bzw} .20$ mm. Mit der Quadrupoloptik QO können die Ionen abgelenkt oder auf den Kondensatoreintrittsspalt $\mathrm{SP}_{1}$ fokussiert werden.

Die durch $\mathrm{SP}_{1}$ gelangenden Ionen werden durch den $90^{\circ}$-Zylinderkondensator $\mathrm{K}_{1}, \mathrm{~K}_{\mathbf{2}}$ auf den Austrittsspalt $\mathrm{SP}_{2}$ fokussiert. Der Kondensator hat einen mittleren Radius von $140 \mathrm{~mm}$. Die Platten $\mathrm{K}_{1}$ und $\mathrm{K}_{2}$ bestehen aus Messing und sind auf der dem Strahl zugewandten Seite präzise gedreht und poliert sowie überall vernickelt. Die Höhe des Kondensators ist mit $100 \mathrm{~mm}$ reichlich bemessen, um Randfeldeffekte auszuschließen, der Plattenabstand ist groß gewählt $(20 \mathrm{~mm})$, so daß

11 R. Herzog, Z. Phys. 97, 596 [1935].
Justierfehler sowie eventuelle lokale Aufladungen der Platten weniger stören. Die sogenannten „HERzoG-Blenden" ${ }^{11} \mathrm{H}$ liegen auf Erdpotential und beschneiden das Randfeld. $\mathrm{J}_{1}, \mathrm{~J}_{2}$ sind Justierblenden zum Schwenken des Strahls in der Horizontalen. In der Vertikalen kann der Strahl mittels der Blenden $\mathrm{B}_{1}-\mathrm{B}_{8}, \mathrm{~L}_{1}-\mathrm{L}_{4}$ geschwenkt und fokussiert werden; die je 3 Blendenpaare $\mathrm{B}_{1}\left(\mathrm{~B}_{2}\right), \mathrm{L}_{1}\left(\mathrm{~L}_{2}\right)$ usw. vor und hinter dem Kondensator wirken als eindimensionale, in der Vertikalen fokussierende Linsen.

$\mathrm{SP}_{2}$ ist zugleich der Eintrittsspalt des Massenspektrometers. Es ist ein magnetisches $60^{\circ}$.Spektrometer mit $20 \mathrm{~cm}$ Bahnradius, die Krümmung ist der im Kondensator entgegengesetzt. Hinter dem Spektrometeraustrittsspalt $\mathrm{SP}_{3}$ ist ein 17-stufiger Cu-Be-Ionenmultiplier angeordnet. Die Weiten der Spalte waren: $\mathrm{SP}_{1} 0,7_{2} \mathrm{~mm}$; $\mathrm{SP}_{2} 0,2_{6} \mathrm{~mm} ; \mathrm{SP}_{3} 0,6_{3} \mathrm{~mm}$. Damit wurde eine Massenauflösung von 200 erreicht (Halbwertsbreite).

Die aus hartverlötetem Messing bestehende Vakuumapparatur wird durch eine Quecksilberdiffusionspumpe $(600 \mathrm{l} / \mathrm{sec})$ ausgepumpt. Vor jeder Inbetriebnahme wurden grundsätzlich zuerst die ausheizbaren Teile KT, $\mathrm{EP}, \mathrm{SP}_{1}, \mathrm{SP}_{2}, \mathrm{~K}_{1}$ und $\mathrm{K}_{2}$ auf ihre Endtemperatur gebracht sowie die äußere Heizung des Massenspektrometerrohres eingeschaltet. Alle Heizungen blieben während der Messungen in Betrieb, ausgenommen die von KT, da hier die Heizung durch die Kathode ausreichte. Das Aufheizen vor Beginn der Messung ist wesentlich. Es hatte sich nämlich verschiedentlich gezeigt, daß durch 
Beschuß von kalten Oberflächen mit geladenen Teilchen isolierende Schichten entstehen, die auch durch nachträgliches sehr hohes Ausheizen nicht entfernt oder leitend gemacht werden können. Auf einer warmen Oberfläche gebildete Schichten sind dagegen offenbar von vornherein viel besser leitend (vgl. auch ${ }^{12}$ ). Der Untergrunddruck bei heißer Apparatur lag meist bei ca. $1,5 \cdot 10^{-7}$ Torr. Der Einlaß von nicht ausfrierbaren Gasen ergab maximal einen Anstieg auf das Doppelte, bei ausfrierbaren Gasen war er unmerklich.

An den Multiplierausgang können Widerstände von $10^{8}$ bis $10^{11} \mathrm{Ohm}$ angeschlossen werden. Die an ihnen entstehende Spannung wird über einen Schwingkondensatorverstärker einem Schreiber zugeführt.

Die Verstärkung des Multipliers bei $2,4 \mathrm{kV}$ dürfte bei den meisten Messungen etwa $10^{5}$ betragen haben. Unter dieser Annahme lagen die gemessenen Ionenströme zwischen etwa $10^{-13}$ und $10^{-19} \mathrm{~A}$.

Abb. 3 zeigt die Spannungsversorgung der Ionenquelle. Sie besteht aus fünf elektronisch stabilisierten Gleichspannungsnetzgeräten und einer Anodenbatterie

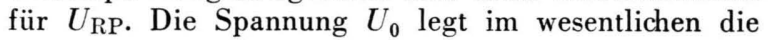

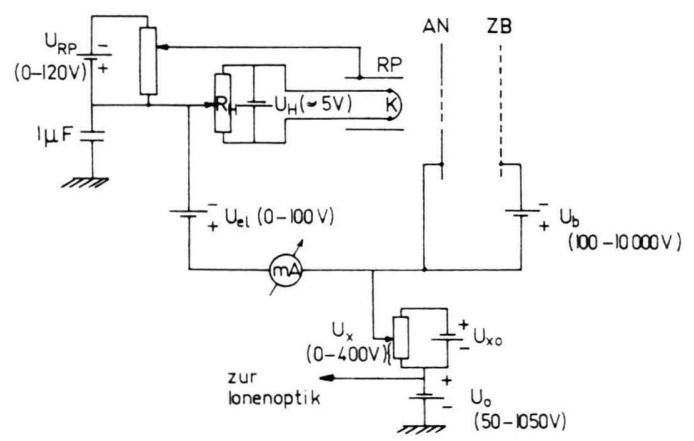

Abb. 3. Schema der Spannungsversorgung.

Energie fest, mit der die Ionen durch Kondensator und Spektrometer fliegen sollen. $\mathrm{Zu}$ ihr addiert sich die Spannung $U_{\mathrm{x}}$, die die unabhängige Variable der ganzen Messung darstellt. $U_{\mathrm{x}}$ wird an einem motorgetriebenen Potentiometer abgegriffen. Dieses ist ein auf $0,1 \%$ lineares Helipot von $300 \mathrm{kOhm}( \pm 1 \%)$, durch das ein Strom von $1 / 3 \mathrm{~mA} \pm 1 \%$ fließt. Die meistens benutzte Geschwindigkeit war $0,0125 \mathrm{U} / \mathrm{sec} \triangleq 0,125 \mathrm{~V} / \mathrm{sec}$ für „Schwanzmessungen“, $0,03 \mathrm{~V} / \mathrm{sec}$ für Messungen des „Nebenpeaks“ (s. u.).

$U_{\mathrm{b}}$ ist die Ionenbeschleunigungsspannung. Die Hochspannung ist auf $0,1 \%$ stabilisiert und auf $5 \%$ genau gemessen. Die Elektronenbeschleunigungsspannung $U_{\mathrm{el}}$ wird der Kathodenmitte zugeführt. Der $1 \mu \mathrm{F}$-Kondensator dient zur Unterdrückung von $50 \mathrm{~Hz}$-Brumm auf den Ionenquellenelektroden.

\section{Einzelheiten der Meßkurven und Auswerte- verfahren}

Die Elektronenenergie möge einen festen Wert $U_{\text {el }}$ haben. Der Kondensator sei auf eine feste Ionen- energie $e U_{\mathrm{c}}$ eingestellt. Der Magnetstrom sei so gewählt, daß die gewünschte Masse bei dieser Energie $e U_{\mathrm{c}}$ auf den Auffänger gelangt.

Zunächst werde die Messung der Mutterionen betrachtet. $U_{\mathrm{x}}$ sei zu Beginn so klein, daß das Anodenpotential kleiner als $U_{\mathrm{c}}$ ist. Dann gibt es in der Ionenquelle keinen Punkt, von dem aus Ionen durch den Kondensator gelangen könnten, der Strom ist Null. Sobald mit steigendem $U_{\mathrm{x}}$ das Anodenpotential den Wert $U_{\mathrm{c}}$ erreicht, gelangen die im Gasuntergrund zwischen Anode und Strahl dicht an der Anode gebildeten Ionen zur Messung, der Strom zeigt eine Stufe. Bei einer Ionenziehfeldstärke $|\mathcal{E}|$ und einem Abstand $a$ zwischen Anode und Strahl gelangt $|\mathfrak{F}| \cdot a$ Volt nach der Stufe der Strahl in das „Gesichtsfeld“ des Kondensators, der Strom durchläuft ein hohes Maximum. Danach fällt er wieder auf das Untergrundplateau und nimmt dann allmählich ab, da die jetzt zur Messung gelangenden Ionen von Elektronen gebildet worden sind, die immer weniger Energie haben. Sobald man sich um $U_{\mathrm{el}}-$ I.P. Volt (I.P. = Ionisierungsenergie des Gases) von der „Anodenstufe" entfernt hat, wird der Strom Null.

Für Fragmentionen sind Anodenstufe und Strahlmaximum ebenso vorhanden. Auf der negativen Seite des Strahls findet man wieder ein Stück weit den direkt ionisierten und dissoziierten Untergrund, außerdem kommen hier aber an jeder Stelle die Beiträge von verzögerten Dissoziationen an jeweils einer weiter im Negativen gelegenen Stelle hinzu. Insbesondere findet man dadurch noch Ionen in Gebieten, in die die Elektronen nicht mehr gelangen. Für Fragmentionen schließt sich also an das Strahlmaximum nach der negativen Seite hin ein Schwanz an. Wird er mit großer Verstärkung sorgfältig gemessen, so kann aus der Intensität an einer bestimm. ten Stelle die zwischen einem gewissen $t$ und $t+\mathrm{d} t$ gebildete $\mathrm{Zahl}$ von Fragmentionen gefunden werden. Zur Illustration dient Abb. 4. Sie zeigt die Strahlpeaks des Fragments der Masse 43 von Heptan (Abb. 4 a) sowie der Muttermasse 100 (Abb. 4 b). Der Anfang des Schwanzes bei Masse 43 ist deutlich zu sehen, während rechts von dem Peak in Abb. $4 \mathrm{~b}$ die Intensität auf Null geht. I.P. bezeichnet den Punkt, bis zu dem die Elektronen ionisieren können. Selbst mit größter Verstärkung sind rechts von I.P. keine Ionen $100^{+}$mehr nachzuweisen. Eine eindeutige Zuordnung der bei einem gewissen $\Delta U$ gemes-

12 L. Holland, L. Laurenson u. C. Priestland, Rev. Sci. Instruments 34, 377 [1963]. 


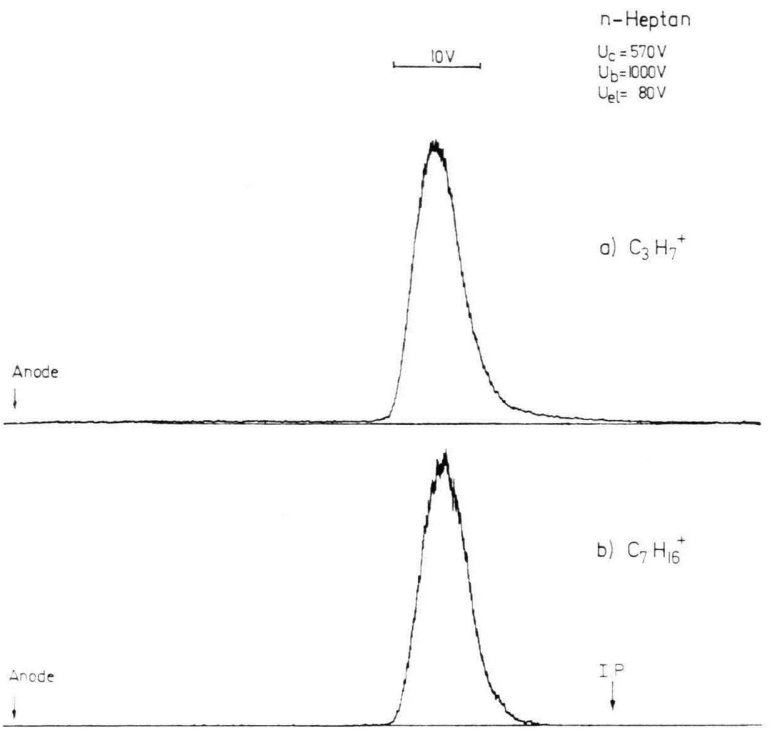

Abb. 4. Beispiele für den Strahlpeak eines Fragments (a) und der Muttermasse (b). Der Schwanz des Peak (a) besteht aus Fragmentionen, die mit einer Zeitverzögerung gebildet wurden. I.P. bezeichnet den Punkt, bis zu dem die Elektronen, von der Anode links her kommend, ionisieren können.

senen Ionenintensität zu einer Zerfallszeit $t$ gemäß (1) ist nur möglich, solange die Zerfallsorte der Ionen im Ionenbeschleunigungsfeld bei positivem Potential liegen (s. Abb. 1). Dagegen gibt es im Potential-,,Tal“ jeweils zwei Zerfallsorte, die zum gleichen $\Delta U$ führen. Der dazugehörige Bereich der Meßkurve ist wegen dieser Doppeldeutigkeit i. allg. nicht auswertbar. Interessant sind die noch später erfolgenden Zerfälle iv dem $1.5 \mathrm{~cm}$ langen feldfreien Raum zwischen Ionenqu. lle und Kondensatoranfang. Sie führen alle zum gleichen $\Delta U$. Alle auf dieser Laufstrecke gebildeten J'rag i, entionen werden daher in einem sogenannten ,. Nebenpeak" an einer bestimmten Stelle der Məßkurve aufintegriert gemessen, und zwar dann, wenn das Strahlpotential $m_{0} / m \cdot U_{\mathrm{c}}$ beträgt. Bei der kontinuierlichen Erhöhung des Ionenquellenpotentials im Verlauf der Messung folgen also aufeinander der Strahlpeak (Potential des Molekularstrahls $=U_{\mathrm{c}}$ ), der Schwanz, der Nebenpeak $\quad\left(\right.$ Strahlpotential $\left.=m_{0} / m \cdot U_{\mathrm{c}}=U_{\mathrm{c}}+\Delta U_{\mathrm{NP}}\right)$ und der doppeldeutige Teil des Schwanzes.

Um auch längere Zerfallszeiten messen zu können, wurden oft auch kleine Spannungen $U_{\mathrm{b}}$ (100 oder $200 \mathrm{~V}$ ) verwendet, bei unverändertem $U_{\mathrm{c}}$. Dann wird aus dem Bremsfeld ein Beschleunigungsfeld.

Zur Auswertung einer Meßkurve $i_{\text {ion }}(\Delta U)$ wurde zunächst durch die Schreiberkurve eine das Rauschen ausgleichende Linie gezogen, wobei versucht werden mußte, weder zuviel Struktur als echt anzuerkennen noch wirklich vorhandene „wegzuglätten“. Dann wurde an willkürlich ausgewählten Stellen $\Delta U$ der Ausschlag abgelesen.

Nachdem man gemäß (1) die Abszisse der Meßkurve von $\Delta U$ auf $t$ umgerechnet hat, muß noch die Ordinate $i_{\text {ion }}$ mit einer Korrekturfunktion multipliziert werden. Der Kondensator erfaßt ja bei jedem $\Delta U$ Ionen aus einem gewissen konstanten Potentialbereich $\mathrm{d} \Delta U$ entsprechend seiner Auflösung. Die gesuchte Größe ist aber die pro Zeitintervall $d t$ gebildete Ionenzahl. Man muß daher noch den gemessenen Ionenstrom mit $\left|\frac{\mathrm{d} \Delta U}{\mathrm{~d} t}\right|=f(\Delta U)$ multiplizieren.

Der direkt dissoziierte Untergrund, der sich auf beiden Seiten an den Strahl anschließt, beeinflußt die Auswertung wesentlich. Obwohl er nur eine Intensität von höchstens einigen \% der Peakhöhe hatte, war doch $u$. U. die gesamte ionisierte Gasmenge aus dem Untergrund mit der aus dem Strahl vergleichbar, nämlich dann, wenn der Abstand Anode - Strahl groß gegen die Strahlbreite war. Dann ist der „Schwerpunkt“ des Strahls, von dem aus die $\Delta U$. Zählung beginnt, nicht mehr gut definiert. Dies ist der Grund, warum die Anode möglichst nahe an den Strahl gelegt wurde. Auf der negativen Seite des Strahls störte der Untergrund noch mehr, da er ähnlich groß wie die zu messende, ihm überlagerte Schwanzintensität war. Die einzige Abhilfe war, die Elektronenenergie so klein zu machen, daß der Strahl gerade noch genügend stark ionisiert wurde, und die Auswertung erst hinter I.P. (Abb. 4) beginnen zu lassen.

Je größer $U_{\mathrm{b}}$ ist, um so breiter erscheint der Strahl auf der Potentialskala. Es wurde aber bei den Messungen bis zu den kleinsten benutzten $\Delta U$ die Strahlform nicht explizit berücksichtigt; der Fehler, den man durch die Ersetzung des wirklichen Strahls durch einen unendlich schmalen Strahl gleicher Fläche im Schwerpunkt des wirklichen macht, wurde in einem der kritischsten Fälle abgeschätzt. Die erste ausgewertete Meßstelle wurde in einem Abstand von der Mitte des Strahlprofils angenommen, der etwa gleich dessen Halbwertsbreite war. Explizite Berücksichtigung der Strahlform führte selbst in diesem extremen Fall nur zu einer um 20\% kürzeren Zerfallszeit als die Ersetzung des Strahls durch eine $\delta$-Funktion in seinem Schwerpunkt. 
Um die mit verschiedenen Werten von $U_{\mathrm{b}}$ aufgenommenen Zerfallskurven, die verschiedene Zeitbereiche überdecken, miteinander vergleichen und aneinander anschließen zu können, wurden sie auf die Fläche des Strahlprofils der Fragmentmasse normiert, so daß die Zerfallskurven die Ordinateneinheit „\% vom Strahl/sec" haben. Eine Trennung von „Strahl“ und „Schwanz“ ist natürlich etwas willkürlich, ließ sich aber meist gut abschätzen. Da die Strahlfläche, ein Maß für die in unmeßbar kurzer Zeit gebildeten Ionen, selbst von $U_{\mathrm{b}}$ abhängt, entsteht dadurch ein systematischer Fehler: Die Meßkurven sind etwas zu steil, das Verhältnis der Ordinaten an ihren beiden Enden ist um $2-30 \%$ zu groß.

Bei der Messung der Nebenpeaks zeigte sich ein überraschender Effekt. Fast alle waren sie nicht einfach Abbilder des Strahlpeaks im Potentialmaßstab $1: 1$, sondern sie waren meist wesentlich breiter als der Strahlpeak. Dies liegt daran, daß bei der Dissoziation eine Zerfallsenergie $E_{\mathrm{z}}$ freigesetzt wird. Ist das dissoziierende Mutterion in Ruhe, so entfallen davon auf die Fragmente $m^{+}$und $\Delta m$ die Anteile

$$
\frac{\Delta m}{m_{0}} \cdot E_{\mathrm{z}} \quad \text { bzw. } \quad \frac{m}{m_{0}} \cdot E_{\mathrm{z}} .
$$

Es ist bekannt, daß bei organischen Fragmentionen die Energien $\Delta m / m_{0} \cdot E_{\mathrm{z}}$ meist unter $1 \mathrm{eV}$ liegen ${ }^{13}$. Die beobachteten Peakverbreiterungen betrugen aber oft $\pm 10 \mathrm{~V}$ und mehr. Das kommt daher, daß die Nebenpeaks von im Fluge dissoziierenden Mutterionen herrühren. Hat nämlich $m_{0}{ }^{+}$im Moment seines Zerfalles eine kinetische Energie $E_{0}$ und wird im Schwerpunktsystem die Zerfallsenergie $E_{\mathrm{z}}$ freigesetzt, so addieren sich die Geschwindigkeiten $v_{\mathrm{z}}$ und $v_{0}$ (nicht die Energien!), die zu den Energien (2 a) und $E_{0}$ gehören. Aus den Geschwindigkeiten

$$
v_{+}=v_{0}+v_{\mathrm{z}} \quad \text { und } \quad v_{-}=v_{0}-v_{\mathrm{z}}
$$

der nach vorwärts bzw. rückwärts abgespaltenen Fragmentionen ergeben sich dann ihre Energien $E_{+}$ und $E_{-}$zu

$$
E_{ \pm}=\frac{1}{m_{0}}\left(\sqrt{m E_{0}} \pm \sqrt{\Delta m E_{z}}\right)^{2} .
$$

Betrachtet man nur Zerfälle nach vorn und hinten und nur eine feste Zerfallsenergie $E_{z}$, so erhält man zwei Gruppen von Fragmentionen aus dem feldfreien Laufraum zwischen Ionenquelle und Kondensator mit den diskreten kinetischen Energien $E_{+}$und

13 R. Fuchs u. R. Taubert, Z. Naturforschg. 19 a, 494 [1964].
$E_{-}$: Der Nebenpeak müßte in zwei Peaks aufspalten. Deren Abstand $\delta U$ auf der Potentialskala ist gegeben durch die Differenz der Ionenquellenpotentiale, die man braucht, um einmal die "Vorwärts-“ und einmal die „Rückwärts“-Ionen durch den Kondensator gelangen zu lassen. Man erhält

$$
e . \delta U=\frac{4}{m} \sqrt{m_{0} \Delta m E_{\mathrm{z}} e U_{\mathrm{c}}} .
$$

Wegen des Faktors $U_{\mathrm{c}}$ ist $e \delta U$ i. allg. viel größer als $E_{z}$, die Anfangsenergien erscheinen „verstärkt".

Da es in Wirklichkeit eine ganze Verteilung von $E_{\mathrm{z}}$ gibt, erhält man nicht einen aufgespaltenen, sondern einen verbreiterten Nebenpeak. Bezeichnet man seine Halbwertsbreite mit $\Gamma$, so ist sie ein Maß für die wesentlichen Anteile der $E_{\mathrm{z}}$-Verteilung:

$$
E_{\mathrm{z}}=\frac{\Gamma^{2}}{16} \frac{m^{2}}{m_{0} \Delta m} \frac{1}{e U_{\mathrm{c}}} .
$$

Es ist zu betonen, daß in dieser Arbeit $E_{\mathrm{z}}$ immer die gesamte im Zerfall freigesetzte Energie ist (auch Übergangsenergie genannt).

Die Berücksichtigung der Winkelverteilung der Zerfälle ergibt keine wesentliche Modifizierung der Peakform.

Die Unschärfe der Energiemessung infolge der Anfangsenergie gilt natürlich auch für die Messung des Schwanzes. Sie bewirkt dort eine Verschlechterung der Zeitauflösung. Für alle Zerfälle, deren Lebensdauerkurven gemessen wurden, war $E_{\mathrm{z}}$ in der Größenordnung $30 \mathrm{mV}$ (außer für $\mathrm{CO}_{2}$, s. u.). Damit erhält man Fehler der Zeitmessung von höchstens einigen Prozent.

Die Messungen dürfen nicht bis zu nahe an den Nebenpeak heran ausgewertet werden, weil seine Ausläufer dort die Intensität wieder ansteigen lassen, ohne daß man es der Meßkurve direkt ansehen kann.

\section{Die Eigenschaften der kritischen Anordnungen in der Apparatur}

\section{Der Molekularstrahl}

Der Druck vor dem ersten Spalt betrug meist ca. 0,1 Torr. Dabei stellte sich zwischen den Spalten für nicht ausfrierbare Gase ein Druck von ca. $3 \cdot 10^{-5}$ Torr ein, für ausfrierbare Gase $3 \cdot 10^{-6}$ Torr. Der Druck im Strahl am Ionisierungsort wurde dadurch bestimmt, $\mathrm{da} \beta$ in die gesamte Apparatur Gas mit einem solchen Druck eingelassen wurde, da $\beta$ die damit gemessene Ionenintensität der normalerweise mit dem Strahl gemessenen gleich wurde. Danach betrug der Druck im 
Strahl ca. $10^{-5}$ Torr. Das Druckverhältnis zwischen Strahl und Untergrund neben dem Strahl lag zwischen 60 und 90. Der Untergrund entsteht dabei nicht durch Diffusion aus dem Raum zwischen den Spalten, sondern aus dem Strahl selbst. Das Strahlprofil kann bequem durch die Abhängigkeit des Ionenstromes vom Ionenquellenpotential gemessen werden (s. Abb. $4 \mathrm{~b}$ ), vorausgesetzt, da $\beta U_{\mathrm{b}}$ so hoch ist, da $\beta$ die Breite des Kondensatordurchlaßbereiches gegen die Strahlbreite auf der Potentialskala vernachlässigbar ist. Die Halbwertsbreite des Strahles betrug meist $60 \mu$ (rein geometrisch waren $40 \mu$ zu erwarten), minimal wurden $28 \mu$ erreicht.

Abb. 5 zeigt eine berechnete Verteilung der Strahlintensität über die Strahlebene, normiert auf den Wert 1 für das Zentrum der Ringspalte.

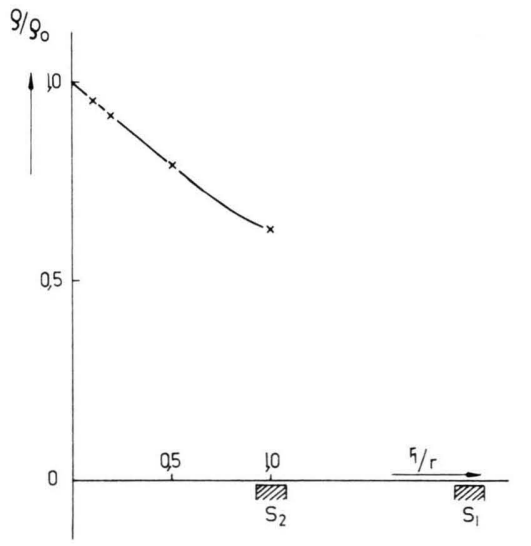

Abb. 5. Verteilung der Molekularstrahlintensität über die Strahlebene.

\section{Der Elektronenstrahl}

Zur Prüfung der Form des Elektronenstrahls wurde bei abgestelltem Molekularstrahl Krypton von etwa $3 \cdot 10^{-7}$ Torr in die gesamte Apparatur eingelassen. Dann wurde bei einer Elektronenenergie von $70 \mathrm{eV}$ wie üblich die Ionenenergieverteilung gemessen, und zwar einmal bei $U_{\mathrm{b}}=100 \mathrm{~V}$ und einmal bei $1000 \mathrm{~V}$.

Die letztere Meßkurve stimmte sehr gut mit den Kurven überein, die EngLander-Golden und RAPP ${ }^{14}$ für die Abhängigkeit des Ionisationsquerschnittes von $U_{\mathrm{el}}$ angeben; die Apparatur ist also auch geeignet, solche relativen Querschnitte zu messen. Die Ionenenergieverteilung bei $U_{\mathrm{b}}=100 \mathrm{~V}$ zeigt dagegen mit zunehmendem Abstand von der Anode einen zu starken Abfall. Dies rührt vom Auseinanderlaufen des Elektronenstrahles her: Bei $U_{\mathrm{b}}=100 \mathrm{~V}$ laufen Elektronen von $70 \mathrm{~V}$ fast durch den ganzen Ionenbeschleunigungsraum, während sie bei $U_{\mathrm{b}}=1000 \mathrm{~V}$ nur $0,7 \mathrm{~mm}$ weit eintauchen. Auf dem längeren Weg läuft das Bündel mehr auseinander, Ionen werden aber nur aus dem Gebiet in der Nähe seiner Achse gesammelt. - Die zu fordernde Homogenität des Elektronenstrahls über die Dicke des Molekularstrahls war hingegen in allen Fällen gewährleistet.

14 P. Englander-Golden u. D. Rapp, Lockheed-Report LMSC 6-74-64-12.

\section{Das Ionenziehfeld}

Störungen der Feldhomogenität können auftreten a) durch die $1 \mathrm{~mm}$ breiten Spaltbacken des inneren Spaltes und Verbiegungen der Anode, b) durch den Durchgriff des Kathodenpotentials durch die Löcher $(0,1 \mathrm{~mm} \phi)$ in $\mathrm{AN}, \mathrm{c})$ durch Raumladung.

Die gute Halbwertsbreite des gemessenen Strahlprofils selbst zeigt, daß a) über das kleine Ionenabzugsgebiet von $8 \mathrm{~mm} \phi$ nicht stört; b) wurde öfter beobachtet und verzerrte das gemessene Strahlprofil in den Fällen, in denen die Anode bis in den Strahl hineinragte. Eine Potentialabschätzung zeigte aber, daß beide Kanten des Strahlprofils für einen Bruchteil des Strahls, nämlich den vor den Löchern, um etwa den gleichen Betrag ins Negative geschoben wurden, so daß die Feldstärke sich kaum änderte. c) Abschätzungen der Raumladung zeigten, daß bei den benutzten Stromdichten von 0,1 bis $0,5 \mathrm{~mA} / \mathrm{cm}^{2}$ noch keine störende Feldverzerrung zu erwarten ist.

\section{Der Zylinderkondensator}

Die Auflösung des Kondensators wurde geprüft mittels Alkali-Ionen, die mit sehr großer Intensität und praktisch monoenergetisch an der heißen Anode durch Oberflächenionisation gebildet werden. Für Ionenenergien $e U_{\mathrm{c}}$ zwischen 340 und $850 \mathrm{~V}$ ergab sich eine Auflösung von ca. 300 (Halbwertsbreite zugrunde gelegt).

Bei der Messung kurzer Zeiten ist es unumgänglich, daß die Ionen beim Durchfliegen von ZB Energien von mehreren $\mathrm{keV}$ haben, die dann (mit der Kombination von variablem Gegenfeld und Kondensator) auf wenige Volt genau gemessen werden müssen. Dabei war zu befürchten, daß die durch den großen Feldstärkesprung an ZB entstehenden starken Feldinhomogenitäten in den Netzmaschen den Ionen so große Querimpulse erteilen, daß die Intensität der nach $10 \mathrm{~cm}$ Laufstrecke durch $\mathrm{SP}_{1}$ gelangenden Ionen stark abnimmt. Abb. 6 zeigt eine Serie von Peaks, die mit gleichem $U_{\mathrm{c}}=341 \mathrm{~V}$, aber verschiedenem $U_{\mathrm{b}}(100,1000$ und $8600 \mathrm{~V})$ un-

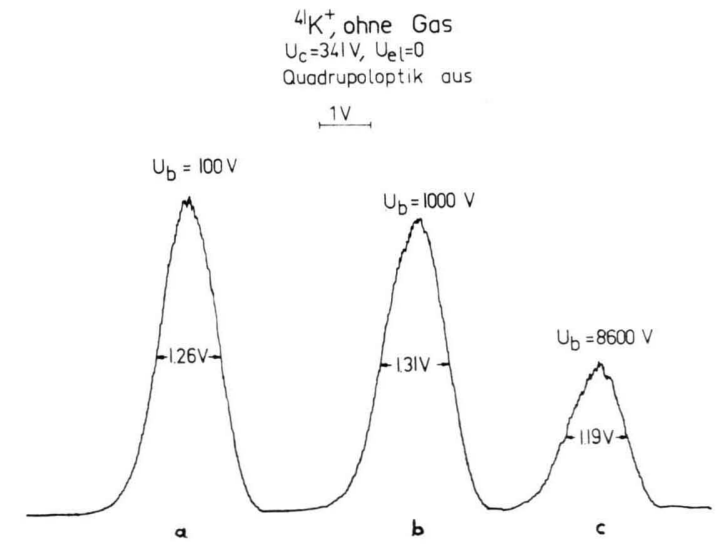

Abb. 6. Die Auflösung und Transmission des Energiekondensators für verschiedene Ionenenergien am Eingang des Gegenfeldes. 
mittelbar nacheinander aufgenommen wurden. Die Empfindlichkeit und Justierung sind für alle drei Peaks dieselben. Hervorzuheben ist, daß bei diesen Messungen die Quadrupoloptik abgeschaltet war, so daß sich Ionenverluste durch Streuung im Netz ZB voll auf der Laufstrecke bis zum Spalt $\mathrm{SP}_{1}$ auswirken mußten. Es zeigt sich, daß zwischen 100 und $1000 \mathrm{~V}$ die Intensität nur um ca. $10 \%$ und bis $8600 \mathrm{~V}$ nur um die Hälfte abnimmt. Die „effektive Auflösung“ ( $U_{\mathrm{b}}$ : Halbwertsbreite) der gesamten Energiemeßanordnung beträgt nach Abb. 6 c mindestens 7200, wenn man einen Intensitätsverlust von $50 \%$ in Kauf nimmt. Mit $U_{\mathrm{b}}=9800 \mathrm{~V}$ wurde eine effektive Auflösung von 10000 bei noch durchaus brauchbarer Intensität erreicht.

\section{Meßergebnisse und Diskussion}

\section{Butan}

Als Musterbeispiel für die Methode wurde zuerst der schon mehrfach untersuchte ${ }^{2,7,8}$ verzögerte Zerfall $\mathrm{C}_{4} \mathrm{H}_{10}{ }^{+} \rightarrow \mathrm{C}_{3} \mathrm{H}_{7}{ }^{+}+\mathrm{CH}_{3}$ von $\mathrm{n}$-Butan besonders sorgfältig gemessen, s. Abb. 7 und ${ }^{15}$. Diese Messung diente einerseits zur Erprobung der Apparatur, zeigte andererseits aber auch erstmalig das wichtige und später auch an anderen Substanzen bestätigte Ergebnis, daß die Zerfälle nicht einem einfachen Exponentialgesetz gehorchen, sondern durch ein Gemisch von e-Funktionen zu beschreiben sind, wie es auch die statistische Theorie fordert. - Im Gegensatz zu den meisten später zu besprechenden Messungen wurden hier Zerfälle aus der ganzen Ionenquelle einschließlich des Raumes ZB - EB benutzt, indem $t(\Delta U)$ und die zugehörigen Korrekturfunktionen auch für diesen Raum mit der veränderlichen Feldstärke berechnet wurden. Dadurch wurden die mit jedem $U_{\mathrm{b}}$ überstrichenen Zeitbereiche größer; sie sind in Abb. 7 unten durch dünne Querlinien für die fünf benutzten Werte von $U_{\mathrm{b}}(100,200,1000,2000,4000 \mathrm{~V})$ angedeutet. An den Enden jedes Bereiches ist durch einen dickeren Balken zugleich die Zeitauflösung angegeben (s. u.). Die fünf Zeitbereiche überlappen sich, z. Tl. dreifach. Es ist bemerkenswert, daß die fünf getrennt normierten Stücke der Meßkurve völlig glatt aneinander anschließen. Da zu einem bestimmten $t$ je nach der Größe von $U_{\mathrm{b}}$ Ionenzerfälle in verschiedenen räumlichen Bereichen der Ionenquelle beitragen, kann man hieraus den wichtigen Schluß ziehen, daß die Nachweiswahrscheinlichkeit für alle zwischen AN und EB gebildeten Ionen dieselbe ist.

Die Zeitauflösung der Messung hängt von $U_{\mathrm{b}}$ und der gerade gemessenen Zeit ab. Nach (1) gilt

$$
\mathrm{d} t \sim \frac{1}{t \cdot U_{\mathrm{b}^{2}}} \mathrm{~d} \Delta U .
$$

Die Unschärfe d $\Delta U$ ist durch die Kombination von Kondensatordurchlaßbreite und geometrischer Strahlbreite gegeben. Die $\mathrm{d} t$ liegen zwischen $10^{-7}$

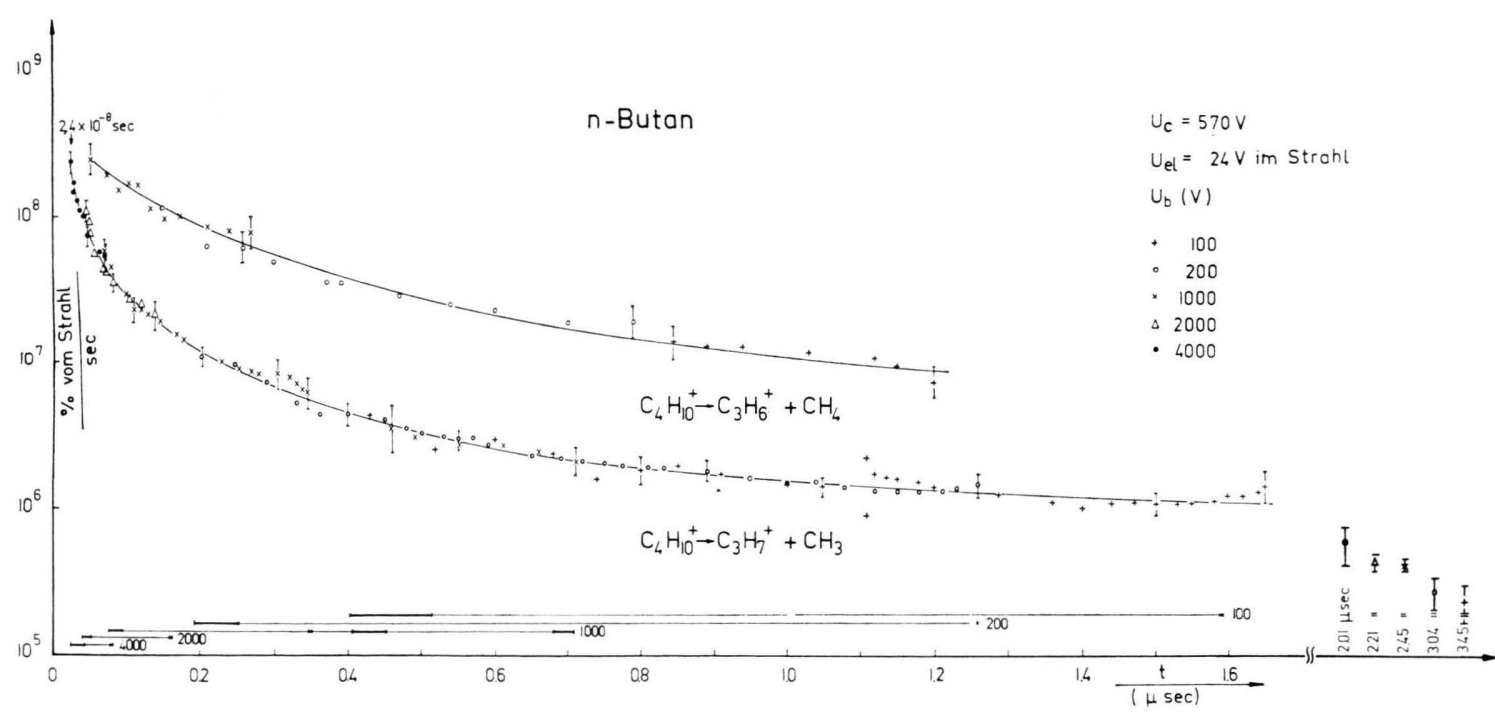

Abb. 7. Differentiell gemessene Zerfallskurven für Butan. Die dünnen Linien am unteren Rand geben den mit der jeweiligen Spannung $U_{\mathrm{b}}$ überstrichenen Zeitbereich an, die dicken Balken an ihren Enden die von $U_{\mathrm{b}}$ und $t$ abhängige Zeitauflösung. Die Kurvenkrümmung entsteht durch Mischung von vielen beteiligten Zerfallskonstanten.

15 O. Osberghaus u. Сh. Ottinger, Phys. Lett. 16, 121 [1965]. 
und $3 \cdot 10^{-9}$ sec und sind i. allg., außer bei den kürzesten Zeiten, so klein gegen $t$ selbst, daß man von einer differentiellen Meßmethode sprechen kann. $\mathrm{Zu}$ sätzlich steht noch die Intensität des Nebenpeaks als ein über die Flugzeit im feldfreien Raum integrierter Wert zur Verfügung. Bei so langen Zeiten ändert sich die Zerfallsrate nur noch wenig, so daß es Sinn hat, die auf die Strahlpeakfläche normierte Nebenpeakfläche durch die Flugzeit zu teilen und diesen Wert als durchschnittliche Zerfallsrate während dieses Fluges bei der Mitte der Flugzeit in Abb. 7 mit einzutragen. So wurden die fünf Punkte in Abb. 7 rechts gewonnen. (Die auf dem Schreiberstreifen gemessene Nebenpeakfläche ist hierfür noch mit dem Faktor $m / m_{0}$ zu korrigieren; da die Energie der Fragmentionen gleich $m / m_{0}$ mal Strahlpotential ist, wird der Nebenpeak nämlich bei gleichmäßiger Variation des Strahlpotentials im Verhältnis $m / m_{0}$ langsamer durchgefahren.) Extrapoliert man die differentiell gemessene Kurve nach langen Zeiten hin und integriert über die Flugzeit im feldfreien Raum, so erhält man Nebenpeakintensitäten, die 1,9-mal so groß wie die beobachteten sind. Dies kommt vermutlich daher, daß die Meßkurve am rechten Ende etwas zu hoch liegt infolge von Beiträgen aus dem Ausläufer des Nebenpeaks, an den diese Messung ziemlich nahe heranführte.

Der jedenfalls recht flache Abfall der Kurve bei langen Zeiten paßt gut zu den Mesungen von von $Z_{\text {AhN }}$ und TAtarczyK ${ }^{4,5}$. Integration der extrapolierten Kurve von 7 bis $101 \mu$ sec liefert als Bildungshäufigkeit von $43^{+}$, bezogen auf die Intensität von $58^{+}$, einen Wert von $1,5 \%$ im Vergleich zu den $4,5 \%$ von ${ }^{4}$.

Die an einigen typischen Stellen in Abb. 7 eingezeichneten Fehlergrenzen geben nur die statistische Unsicherheit an. Sie wurden aus dem Vergleich der zwei bis fünf Messungen abgelesen, aus denen jedes Teilstück der Meßkurve gemittelt wurde.

Eine wichtige Prüfung der Zerfallskurve ist die auf etwaige stoßinduzierte Beiträge. Es ist von den üblichen metastabilen Ionen in Massenspektrometern her bekannt, daß auch an sich stabile Ionen durch Gasstöße in dem feldfreien Raum zur Dissoziation gebracht werden und Metastabile vortäuschen können. Die Prüfung wurde an den bequem meßbaren Nebenpeaks durchgeführt. Bei Druckerhöhung auf

16 W. A. Chupka u. E. Lindnholm, Ark. Fys. 25, 349 [1964].

17 W. A. Chupka u. M. Kaminsky, J. Chem. Phys. 35, 1991 [1961]. das 4 -8-fache blieb die Intensität auf $\pm 10 \%$ gleich. - Eine solche Prüfung wurde bei allen in dieser Arbeit beschriebenen Nebenpeaks außer bei Benzol durchgeführt; in jedem Fall war bei den niedrigsten Drucken der stoßinduzierte Beitrag zur Nebenpeakintensität verschwindend klein.

Die Kurve in Abb. 7 ist mit einer (unkorrigierten) Elektronenergie von $24 \mathrm{eV}$ im Strahl gemessen. Um einen etwaigen Einfluß der Elektronenenergie auf die Häufigkeit der verzögerten Zerfälle festzustellen, wurde die Messung mit nur $14 \mathrm{eV}$ Elektronenenergie zwischen $5 \cdot 10^{-8}$ und $1,4 \cdot 10^{-6}$ sec wiederholt. In diesem ganzen Bereich stimmte die zweite Kurve, die wegen der geringeren Intensität unsicherer ist als die erste, mit dieser auf im Mittel $\pm 20 \%$ überein.

Theoretisch wäre zu erwarten, daß bei Erniedrigung von $U_{\mathrm{el}}$ zuerst die schnellen Zerfälle des Molekülions „aussterben“, die praktisch nur zum Strahlpeak beitragen, so daß die auf diesen normierte Schwanzintensität steigen sollte. Bei noch niedrigerem $U_{\text {el }}$ sollte der Schwanz bei langen Zeiten bevorzugt angehoben werden, so daß die Krümmung abnehmen würde, bis schließlich an der Schwelle der Dissoziation eine Gerade im einfachlogarithmischen Maßstab entstehen sollte. Daß kein Effekt beobachtet wurde, wird dadurch erklärt, daß bei Butan schon $2 \mathrm{~V}$ oberhalb von $\mathrm{AP}\left(\mathrm{C}_{3} \mathrm{H}_{7}{ }^{+}\right)$der Proze $\beta \mathrm{C}_{3} \mathrm{H}_{7} \rightarrow$ $\mathrm{C}_{3} \mathrm{H}_{5}{ }^{+}+\mathrm{H}_{2}$ einsetzt, so daß Masse $43^{+}$sich im Breakdown-Diagramm nur über $2,5-3 \mathrm{~V}$ erstreckt ${ }^{16,17}$. Die oben beschriebene Variation kann sich also nur innerhalb dieses Bereichs abspielen und ist sicher durch die Elektronenenergieinhomogenität noch verschmiert.

Die Kurve für $\mathrm{C}_{3} \mathrm{H}_{6}{ }^{+}$in Abb. 7 beschreibt einen mit $\mathrm{C}_{4} \mathrm{H}_{10}{ }^{+} \rightarrow \mathrm{C}_{3} \mathrm{H}_{7}{ }^{+}+\mathrm{CH}_{3}$ konkurrierenden Primärzerfall des Butanions. Auch dieser verzögerte Prozeß ist schon lange bekannt ${ }^{2,7-9}$. Die Messung wurde mit geringerer Genauigkeit und über einen kürzeren Zeitbereich als die von $\mathrm{C}_{3} \mathrm{H}_{7}{ }^{+}$durchgeführt. Das hauptsächliche Resultat ist, daß die Kurve wieder eine ganz ähnliche Krümmung aufweist, während ihre Intensität, auf die des Strahlpeaks der Masse 42 normiert, etwa siebenmal so gro $\beta$ ist wie bei $43^{+}$. Dies ist in Übereinstimmung mit dem experimentell durch Ladungsaustausch ${ }^{16}$, Elektronensto ${ }^{17}$ und bis $11,9 \mathrm{eV}$ auch durch Photoionisation ${ }^{18}$ gemesse-

18 B. Steiner, C. F. Giese u. M. G. Inghram, J. Chem. Phys. 34, 189 [1961]. 
nen Breakdown-Diagramm von Butan, in dem $42^{+}$ und $43^{+}$einen ähnlichen Einsatz haben, dann aber auseinanderlaufen, indem $42^{+}$bald wieder absinkt, während $43^{+}$zunächst noch viel höher ansteigt; erst bei wesentlich höherer Energie als bei $42^{+}$erfolgt dann der Abfall von $43^{+}$. Gerade dieses obere Gebiet ist aber für den Strahlpeak verantwortlich, der also für $43^{+}$höher, der Schwanz also relativ tiefer liegen sollte als bei $42^{+}$.

Eine Reihe von weiteren verzögert ablaufenden Primär- und Sekundärzerfällen von Butanionen wurde durch die zugehörigen Nebenpeaks nachgewiesen. Die Lage des Nebenpeaks $\Delta U_{\mathrm{NP}}=(\Delta m / m) U_{\mathrm{c}}$ bestimmt ja den Zerfall eindeutig, da $U_{\mathrm{c}}$ (z. B. durch Eichung mit $\mathrm{K}^{+}$-Ionen von der Anode, deren Potential direkt gemessen wird) sowie $m$ aus dem Massenspektrum bekannt sind. Dies ist ein großer Vorteil gegenüber der üblichen Methode der Identifizierung von Metastabilen der scheinbaren Masse $m^{*}=m^{2} / m_{0}$. In Tab. 1 sind die Ergebnise zusammengefaßt.

\begin{tabular}{|c|c|c|c|c|c|c|}
\hline \multirow{2}{*}{$\begin{array}{c}0 \\
\mathrm{Nr} .\end{array}$} & \multirow{2}{*}{$\begin{array}{c}1 \\
\text { Prozeß }\end{array}$} & \multirow{2}{*}{$\begin{array}{c}2 \\
\Delta m\end{array}$} & \multirow{2}{*}{$\begin{array}{c}3 \\
\\
I_{\text {rel }} \\
(\%)\end{array}$} & \multicolumn{3}{|c|}{$\begin{array}{ccc}4 & 5 & 6 \\
I_{\text {metast. }}\left(I_{43}=100\right)\end{array}$} \\
\hline & & & & $\begin{array}{l}\text { diese } \\
\text { Arbeit }\end{array}$ & $\left(\begin{array}{c}\text { Anm } \\
19\end{array}\right)$ & $\left(\begin{array}{l}\text { Anm } \\
20\end{array}\right)$ \\
\hline 1 & $58^{+} \rightarrow 43^{+}+15$ & 14,95 & 0,82 & 0,82 & 0,20 & 0,10 \\
\hline 2 & $58^{+} \rightarrow 42^{+}+16$ & 15,4 & 10,7 & 1,32 & 0,13 & 0,0 \\
\hline 3 & $57^{+} \rightarrow 41^{+}+16$ & 16,1 & 2,76 & 0,78 & - & 0,02 \\
\hline 4 & $43^{+} \rightarrow 41^{+}+2$ & 1,94 & 13,6 & 3,88 & 0,47 & 0,34 \\
\hline 5 & $29^{+} \rightarrow 27^{+}+2$ & 2,01 & 0,80 & 0,3 & 0,12 & 0,07 \\
\hline 6 & $42^{+} \rightarrow 41^{+}+1$ & 0,94 & 0,28 & 0,08 & - & - \\
\hline 7 & $57^{+} \rightarrow 29^{+}+28$ & 28,1 & 0, & 0,0 & - & - \\
\hline 0 & $55^{+} \rightarrow 29^{+}+26$ & 26,1 & 0,05 & 0,02 & - & 0,004 \\
\hline
\end{tabular}

Tab. 1. Verzögerte Dissoziationen bei n-Butan.

Spalte 2 enthält die aus dem gemessenen $\Delta U_{\mathrm{NP}}$ berechneten Werte für $\Delta m$ und gibt ein $\mathrm{Ma}$, wie genau sich die Prozesse identifizieren lassen. Spalte 3 enthält das Verhältnis $\left(m / m_{0}\right.$ mal Nebenpeakfläche) : (Strahlpeakfläche) in $\%$, wobei die Fläche durch Halbwertsbreite mal Höhe angenähert wurde. Spalte 4 geht aus Spalte 3 durch Multiplikation mit $I_{m} / I_{m b}$ aus dem normalen Massenspektrum ${ }^{19}$ hervor, wo $I_{m b}$ die Intensität des stärksten Peaks im Spektrum ist. Damit werden die „Metastabilen"-Intensitäten auf $I_{m_{\mathrm{b}}}=100$ bezogen, wie es üblich ist. Die letzten Spalten geben für diese Größe die anderweitig gemesse-

19 Mass Spectral Data, Amer. Petrol. Inst. Res. Project 44 [1953].

20 H. M. Rosenstock u. C. E. Melton, J. Chem. Phys. 26, 314 [1957]. nen Werte, die allerdings Peakhöhenverhältnisse darstellen.

Weitere Prozesse, nach denen erfolglos gesucht wurde, waren die verzögerte Bildung von $57^{+}, 56^{+}$, $55^{+}$und $15^{+}$(Nachweisgrenze siehe unter Heptan). Die Anordnung erwies sich als recht empfindlich zum Aufsuchen von Metastabilen, so daß auch zwei Prozesse gefunden werden konnten, die in ${ }^{20}$ nicht aufgeführt sind, obwohl dort Vollständigkeit angestrebt wurde. - Auffallend große Metastabilen-Intensitäten haben auch Futrell ${ }^{21}$ unter Benutzung eines doppeltfokussierenden Spektrometers sowie Ferguson u. a. ${ }^{22}$ mit einem Flugzeitspektrometer gefunden.

Das Fragment $41^{+}$ist ein schönes Beispiel für die Identifizierung der Prozesse: Auf Masse 41 wurden längs des Schwanzes drei Nebenpeaks gefunden, deren Abstände vom Strahl sich wie $1: 2$ : 16 verhielten, entsprechend den Prozessen 6, 4 und 3.

\section{Heptan}

Bei einem so großen Molekül wie Heptan wird eine langsame Variation der Zerfallskonstanten $k$ mit der inneren Energie $E$ des Moleküls nach der statistischen Theorie vorausgesagt.

Um zunächst einen Überblick über die vorkommenden verzögerten Dissoziationen zu erhalten, wurde ein „reines Metastabilen-Massenspektrum“ gemessen. Dazu muß man bei kleinem $U_{\text {el }}(30 \mathrm{~V})$ ein $\Delta U$ fest so einstellen, daß mit Sicherheit keine durch direkte Dissoziation gebildeten Fragmente gemessen werden. Dann wird einfach der Magnetstrom sehr langsam variiert, so daß nach und nach alle Fragmente, die verzögert gebildet werden können, einen Peak im Massenspektrum liefern. Abb. 8 zeigt das Ergebnis einer solchen Messung, die insgesamt 100 min dauerte. Fast jeder Peak des normalen Spektrums erscheint hier wieder, ausgenommen u. a. Masse 15 und natürlich der Mutterpeak. Aus den Intensitäten dieses Metastabilen-Spektrums kann man keine direkten Schlüsse ziehen, da zu jeder Masse ein anderer Ausschnitt der Schwanzkurve beiträgt.

Dann wurde zu allen diesen Massen $m$ außer 28 und 40 die Masse $m_{0}$ bestimmt durch Aufsuchen des Nebenpeaks. Die so identifizierten Zerfälle (zusätz-

21 J. H. Futrell, K. R. Ryan u. L. W. Sieck, J. Chem. Phys. 43, 1832 [1965].

22 R. E. Ferguson, K. E. McCulloh u. H. M. Rosenstock, J. Chem. Phys. 42, 100 [1965]. 


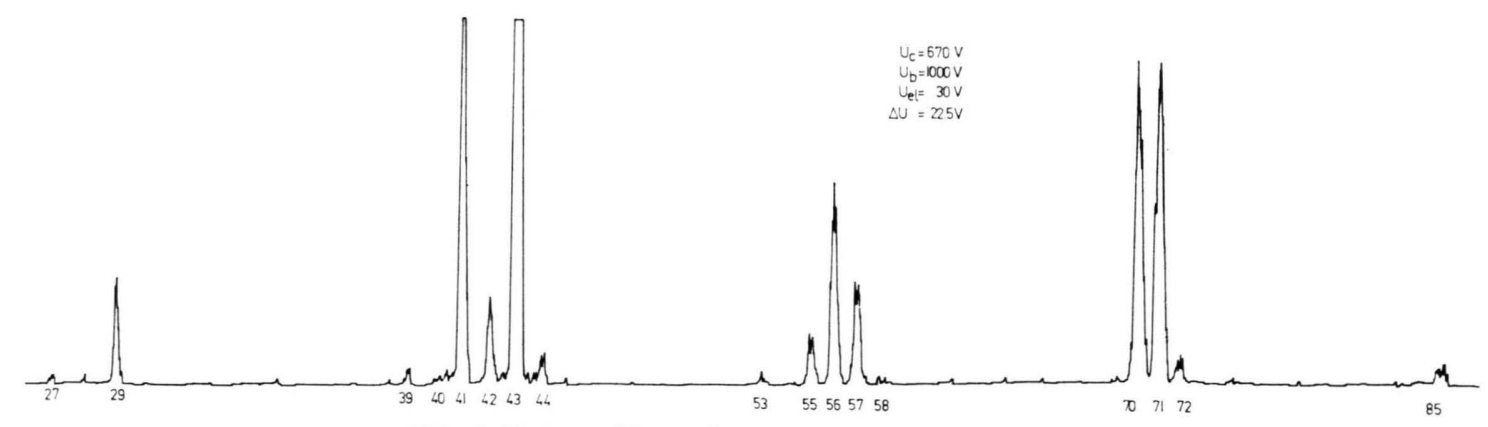

Abb. 8. Reines „Metastabilen-Spektrum“ des Heptans.

\begin{tabular}{|c|c|c|c|c|c|}
\hline 0 & 1 & 2 & $\begin{array}{c}3 \\
I_{\mathrm{rel}}\end{array}$ & $\begin{array}{c}4 \\
I_{\text {metast }}\end{array}$ & $\left(I_{43} \stackrel{5}{=} 100\right)$ \\
\hline Nr. & FTozes & $\Delta m$ & & $\begin{array}{l}\text { diese } \\
\text { Arbeit }\end{array}$ & $\left(\right.$ Anm. $\left.{ }^{19}\right)$ \\
\hline 1 & $55^{+} \rightarrow 53^{+}+2$ & 1,94 & 14,6 & 0,28 & - \\
\hline 2 & $43^{+} \rightarrow 41^{+}+2$ & 2,01 & 5,30 & 2,79 & 0,67 \\
\hline 3 & $41^{+} \rightarrow 39^{+}+2$ & 1,97 & 5,39 & 0,91 & 0,14 \\
\hline 4 & $29^{+} \rightarrow 27^{+}+2$ & 1,97 & 0,43 & 0,16 & 0,10 \\
\hline 5 & $28^{+} \rightarrow 26^{+}+2$ & 2,00 & 0,59 & 0,014 & - \\
\hline 6 & $100^{+} \rightarrow 85^{+}+15$ & 14,7 & 2,52 & 0,048 & - \\
\hline 7 & $70^{+} \rightarrow 55^{+}+15$ & 14,9 & 3,82 & 0,42 & - \\
\hline 8 & $58^{+} \rightarrow 42^{+}+16$ & 15,85 & 0,71 & 0,17 & - \\
\hline 9 & $57^{+} \rightarrow 41^{+}+16$ & 15,7 & 17,1 & 8,95 & 0,13 \\
\hline 10 & $55^{+} \rightarrow 29^{+}+26$ & 26,1 & 0,64 & 0,30 & 0,02 \\
\hline 11 & $53^{+} \rightarrow 27^{+}+26$ & 26,1 & 0,24 & 0,09 & - \\
\hline 12 & $85^{+} \rightarrow 57^{+}+28$ & 27,8 & 0,32 & 0,15 & - \\
\hline 13 & $84^{+} \rightarrow 56^{+}+28$ & 27,8 & 0,09 & 0,023 & - \\
\hline 14 & $71^{+} \rightarrow 43^{+}+28$ & 27,8 & 7,45 & 7,45 & 0,59 \\
\hline 15 & $70^{+} \rightarrow 42^{+}+28$ & 28,0 & 1,03 & 0,25 & - \\
\hline 16 & $57^{+} \rightarrow 29^{+}+28$ & 28,1 & 0,97 & 0,45 & - \\
\hline 17 & $100^{+} \rightarrow 71^{+}+29$ & 28,8 & 2,28 & 1,02 & 0,12 \\
\hline 18 & $100^{+} \rightarrow 70^{+}+30$ & 29,7 & 9,65 & 1,68 & 0,10 \\
\hline 19 & $85^{+} \rightarrow 43^{+}+42$ & 41,7 & 0,25 & 0,25 & 0,02 \\
\hline 20 & $100^{+} \rightarrow 57^{+}+43$ & 42,7 & 0,43 & 0,21 & - \\
\hline 21 & $100^{+} \rightarrow 56^{+}+44$ & 44,2 & 2,95 & 0,79 & $0,03 ?$ \\
\hline 22 & $100^{+} \rightarrow 42^{+}+58$ & 57,9 & 0,30 & 0,07 & - \\
\hline
\end{tabular}

Tab. 2. Verzögerte Dissoziationen bei n-Heptan.

lich wurde noch $28^{+} \rightarrow 26^{+}+2$ gefunden) sind in Tab. 2 zusammengestellt, nach Art von Tab. 1. Nach den folgenden Prozessen wurde ebenfalls gesucht:

$$
\begin{aligned}
& 85^{+} \rightarrow 41^{+}+44 ; 70^{+} \rightarrow 41^{+}+29 ; 55^{+} \rightarrow 27^{+}+28 ; \\
& 57^{+} \rightarrow 27^{+}+30 ; 43^{+} \rightarrow 28^{+}+15 ; 43^{+} \rightarrow 27^{+}+16 ; \\
& 42^{+} \rightarrow 27^{+}+15 ; 41^{+} \rightarrow 26^{+}+15 ; \\
& 42^{+} \rightarrow 41^{+}+1 \text { und } 100^{+} \rightarrow 43^{+}+57 .
\end{aligned}
$$

Für sie wurden keine Nebenpeaks gefunden, d. h. die Peakhöhe ist kleiner als etwa 0,001 , bezogen auf $I_{43}=100$ (ausgenommen $42^{+} \rightarrow 41^{+}+1$, wo die Ausschließungsgrenze wegen der anderen nach $41^{+}$führenden Prozesse nur ca. 0,1 ist). Bei einem Vergleich mit den gefundenen Prozessen in Tab. 2 ist zu be- rücksichtigen, daß die dort aufgeführten Werte Peakflächenverhältnisse sind. Ohne Einrechnung der Peakbreiten, d.h. ausgedrückt durch die Peakhöhenverhältnisse, wären die Werte in Tab. 2, Spalte 4, 2- bis 15-mal kleiner.

Interessant ist der Prozeß 8. Masse 58 ist ein reiner Isotopenpeak, dieser Prozeß ist also wie Nr. 9 $\mathrm{C}_{4} \mathrm{H}_{9}{ }^{+} \rightarrow \mathrm{C}_{3} \mathrm{H}_{5}{ }^{+}+\mathrm{CH}_{4}$, jedoch mit einem ${ }^{13} \mathrm{C}$-Atom in den Ionen. Die Intensitäten der Nebenpeaks (Spalte 4) verhalten sich auch etwa wie $\frac{3}{4} \cdot I_{58}: I_{57}$ $=0,03$ (der Faktor $\frac{3}{4}$ berücksichtigt die statistische Verteilung der C-Atome im ${ }^{13} \mathrm{C}^{12} \mathrm{C}_{3} \mathrm{H}_{9}^{+}$), und vor allem sind die gemessenen Anfangsenergien (siehe Tab. 4) für beide Prozesse aufallend groß und innerhalb der Fehlergrenzen gleich.

Erwähnenswert ist, daß auch Massen wie 53 und besonders 84, die im Spektrum sehr schwach sind ( 1,92 bzw. $0,17 \%$ von $\left.43^{+}\right)$, noch meßbare verzögerte Dissoziationen liefern (Prozeß 11 und 13). Bцоом u. a. ${ }^{23}$ stellten die Regel auf, daß sowohl $m_{0}{ }^{+}$ als auch $\mathrm{m}^{+}$starke Peaks haben müßten, um einen metastabilen Peak $\mathrm{m}^{2} / \mathrm{m}_{0}$ zu ergeben. - Der Prozeß 22 stellt die einzige gefundene Ausnahme von der Stevensonschen Regel dar, nach der i. allg. das geladene Fragment die größere Masse hat. Demgegenüber wurde der Prozeß $100^{+} \rightarrow 43^{+}+57$ nicht gefunden.

Zusammenfassend läßt sich sagen, daß bei verzögerten Dissoziationen im Heptan vorzugsweise Moleküle $\left(\mathrm{H}_{2}, \mathrm{CH}_{4}, \mathrm{C}_{2} \mathrm{H}_{2}, \mathrm{C}_{2} \mathrm{H}_{6}, \mathrm{C}_{3} \mathrm{H}_{6}, \mathrm{C}_{3} \mathrm{H}_{8}, \mathrm{C}_{4} \mathrm{H}_{10}\right)$ abgespalten werden, in einigen $F$ ällen aber auch Radikale (zweimal $\mathrm{CH}_{3}$ und je einmal $\mathrm{C}_{2} \mathrm{H}_{5}$ und $\mathrm{C}_{3} \mathrm{H}_{7}$ ), diese bis auf Nr. 7 alle in Primärprozessen. Es gibt erstaunlich viele (sechs) Primärprozesse. Nach der statistischen Theorie sollte von konkurrierenden Pro-

23 E. G. Bloom, F. L. Mohler, C. E. Wise u. E. J. Wells, NBS RP 2005, 43 [1949]. 
zessen jeweils nur der mit der niedrigsten Aktivierungsenergie auch verzögert ablaufen können ${ }^{24,25}$. Prozesse mit wenig höherer Aktivierungsenergie können nach dieser Theorie nur in besonderen Fällen (vor allem einfache $\mathrm{C}$-C-Brüche) ebenfalls verzögert beobachtbar werden, falls ihr Frequenzfaktor hoch genug ist. Es sind ja auch drei der Primärprozesse $\mathrm{C}$-C-Brüche; vor allem für Prozeß 6, der nach ${ }^{26}$ die höchste Aktivierungsenergie $(0,8 \mathrm{eV})$ braucht, wird dieses Argument gelten. Immerhin mag man in dem Auftreten so vieler verzögerter Primärzerfälle ein Anzeichen dafür sehen, daß die Zerfälle nicht alle aus dem elektronischen Grundzustand erfolgen, sondern daß auch isolierte Elektronenterme beteiligt sind.

Nachdem die Tab. 2 vorlag, wurden die Zerfälle ausgesucht, für die eine Messung der Zerfallsfunktion möglich war. Die Kriterien hierfür sind: 1. $\Delta m / m$ sollte möglichst groß sein, um zwischen Strahl und Nebenpeak genügend Raum zu haben, der frei von direkter Dissoziation und den Nebenpeakausläufern ist. 2. Die Masse $m$ soll nur auf eine Weise gebildet werden können. 3. Die „absolute“ Intensität (Spalte 4) sollte möglichst groß sein. Dann kommen nur die Prozesse 7, 17 und 18 in Frage. Bei zwei weiteren (14 und 21) ist eine Beimischung eines anderen Prozesses (19 bzw. 13) von einigen \% vorhanden, die auf dasselbe $m$ führt. Sie wurden ebenfalls gemessen, zumal alle Kurven, wie sich zeigt, ähnlich verlaufen, also keine Verzerrung zu erwarten ist außer an der Stelle der Nebenpeaks der beigemischten Prozesse (trifft nur auf das Paar 21-13 $\mathrm{zu})$.

In den Abb. 9, 10 und 11 sind die Ergebnisse zusammengestellt. $U_{\text {el }}$ war überall $30 \mathrm{~V}$ im Strahl. Die Mittelwerte aus den Nebenpeaks sind wie in Abb. 7 rechts eingetragen.

Für das intensive Fragment $43^{+}$wurden auch Messungen unter Verwendung des ganz schmalen Molekularstrahls $(28 \mu)$ und der höchsten Ziehfeldstärke $\left(U_{\mathrm{b}}=8600\right.$ und $\left.9800 \mathrm{~V}\right)$ gemacht, um noch möglichst kurze Zerfallszeiten zu erfassen. Trotz der kleinen geometrischen Breite des Strahls erschien das Strahlprofil bei diesen hohen Feldstärken auf der Potentialskala doch noch sehr breit (ca. 25 bis

24 D. P. Stevenson, Structural Interpretation of Spectra, Technical Report No. 51-61 [1961].

25 D. P. Stevenson u. D. O. Schissler, Actions Chimiques et Biologiques des Radiations, Masson et Cie., Paris 1961, S. $230-235$.
$30 \mathrm{~V}$ ); einige Meßpunkte wurden daher mit einer um $20 \mathrm{~V}$ niedrigeren Elektronenenergie gewonnen, so daß die Elektronen noch im Strahl bis unter I.P. abgebremst wurden, was das gemessene Strahlprofil auf der der Anode abgewandten Seite beschnitt. So war es möglich, wenn auch unter starker Intensitätseinbuße, bei der höchsten Feldstärke von $9800 \mathrm{~V} / \mathrm{cm}$ noch bei einem $\Delta U=14 \mathrm{~V}$ zu messen. Dem entspricht eine kürzeste gemessene Zeit von $7,4 \cdot 10^{-9}$ sec. Eine etwaige Veränderung der Zerfallshäufigkeit durch die Erniedrigung von $U_{\text {el }}$ war wiederum nicht festzustellen, die durch das Strahlprofil gegebene Unschärfe von $U_{\mathrm{el}}$ ist aber ja ohnehin in diesem Fall groß.

Die Messung von $43^{+}$in Abb. 10 überdeckt von allen in dieser Arbeit gemessenen Zerfällen das größte Zeitintervall, nämlich eine Zeitvariation um den Faktor 180 für die differentielle Messung, unter Einschluß der gesamten Flugzeit bis zum Kondensator sogar 650 .

Der Versuch einer theoretischen Deutung der Zerfallskurven wird in einer späteren Arbeit gegeben.

\section{Methan und die deuterierten Methane}

Dibeler und Rosenstock ${ }^{27}$ haben eine verzögerte Dissoziation im $\mathrm{CD}_{4}$ untersucht: $\mathrm{CD}_{4}^{+} \rightarrow \mathrm{CD}_{3}{ }^{+}+\mathrm{D}$. Diese Verbindung wurde daher gewählt, um eine Zerfallskurve für ein möglichst kleines Molekül zu messen. Es wurden je zwei bis fünf Messungen mit $U_{\mathrm{b}}=100,200,400,1000,2000,4000 \mathrm{~V}$ gemacht. Die Elektronenenergie im Strahl war in allen Fällen $24 \mathrm{eV}$.

Die Messungen erwiesen sich als sehr schwierig, vor allem wegen der geringen Intensität. Zwischen Strahl und Schwanz bestanden Intensitätsverhältnisse von $10^{5}$ und mehr. Eine weitere Komplikation ergab sich daraus, daß das Gas etwa $4 \% \mathrm{CD}_{3} \mathrm{H}$ enthielt. $\mathrm{CD}_{3} \mathrm{H}$ hat aber eine im Vergleich zu $\mathrm{CD}_{4}$ wesentlich intensivere verzögerte Dissoziation $\mathrm{CD}_{3} \mathrm{H}^{+} \rightarrow \mathrm{CD}_{3}{ }^{+}$ $+\mathrm{H}$, die auch auf Masse 18 führt, aber einen Nebenpeak genau in der Mitte zwischen dem Strahl- und Nebenpeak der Reaktion $\mathrm{CD}_{4}{ }^{+} \rightarrow \mathrm{CD}_{3}{ }^{+}+\mathrm{D}$ liefert. Dieser störte also die Schwanzmessung erheblich. Da dieser "falsche“ Nebenpeak während der Messung nicht deutlich abgegrenzt und so von vornherein

26 B. Brehm, Dissertation, Freiburg 1965.

27 V. H. Dibeler u. H. M. Rosenstock, J. Chem. Phys. 39, 1326 [1963]. 


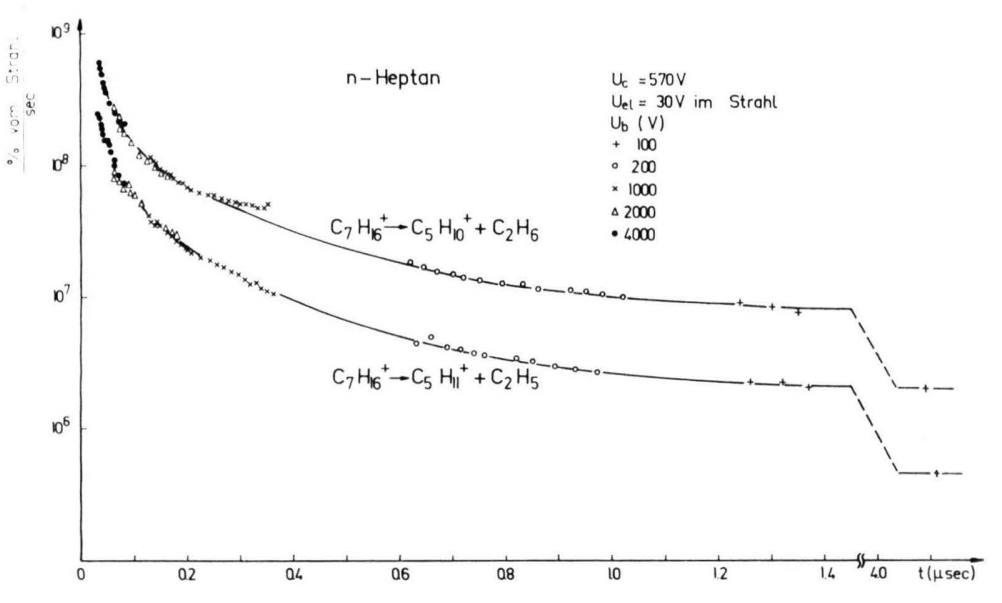

Abb. 9. Zerfallskurven für n-Heptan.

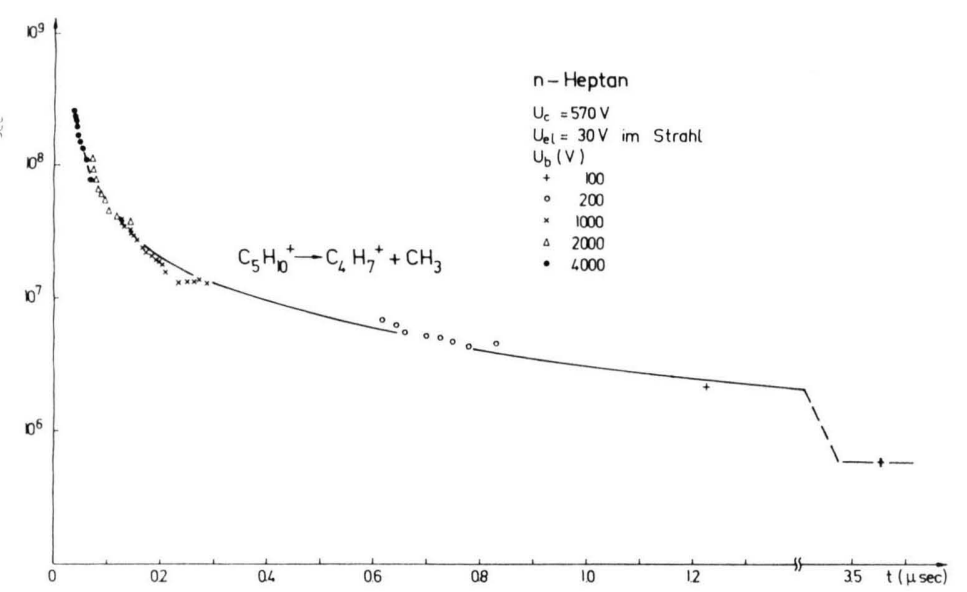

Abb. 11. Zerfallskurve für n-Heptan.

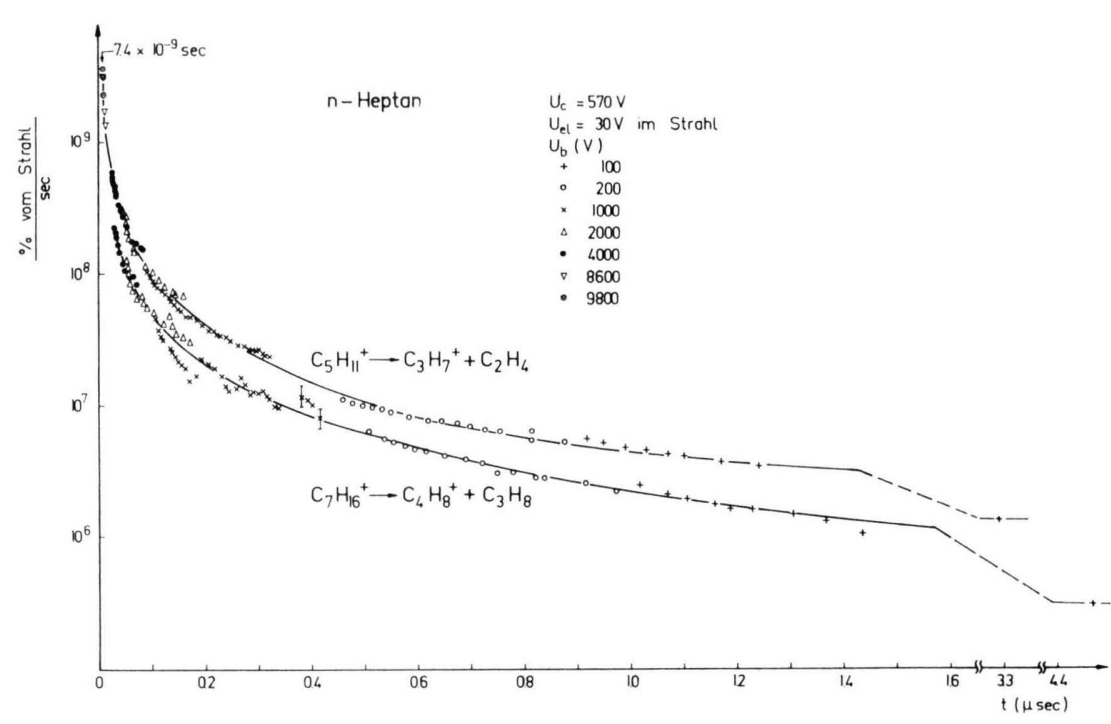

Abb. 10. Zerfallskurven für n-Heptan.

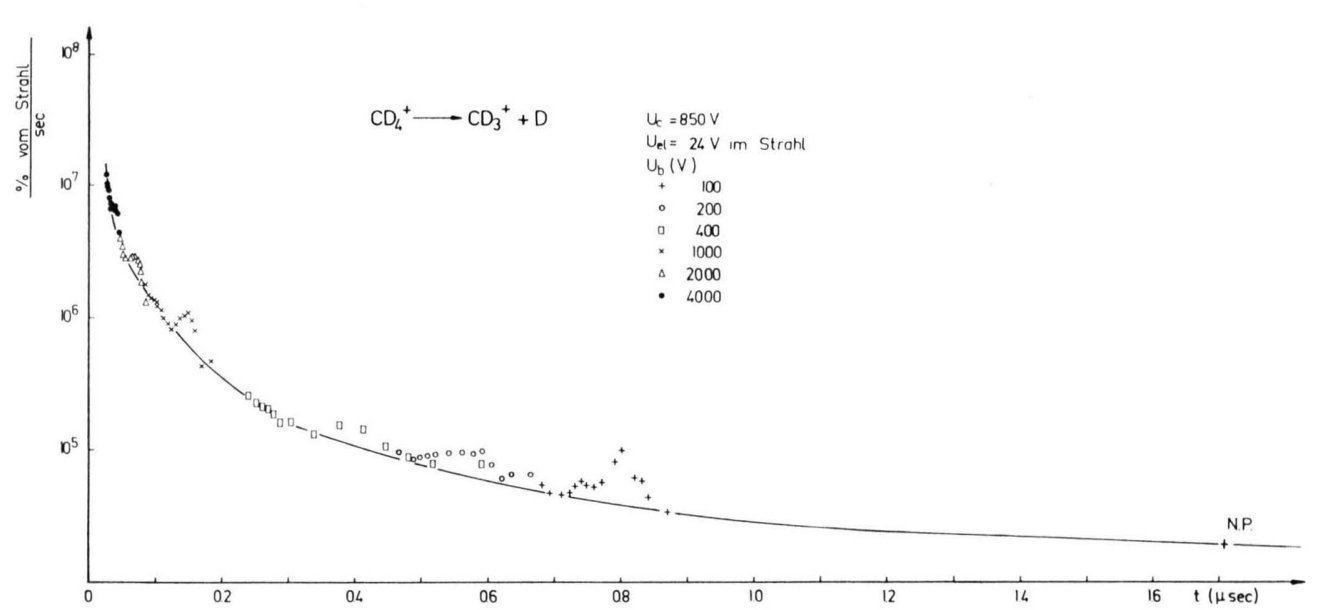

Abb. 12. Zerfallskurve für $\mathrm{CD}_{4}$ mit überlagerten „falschen“ Nebenpeaks aus der Reaktion $\mathrm{CHD}_{3}{ }^{+} \rightarrow \mathrm{CD}_{3}{ }^{+}+\mathrm{H}$. 
weggelassen werden konnte, sind in Abb. 12 zunächst alle abgelesenen Intensitäten eingetragen worden. Dabei wird dieser falsche Nebenpeak erst deutlich sichtbar, und zwar für jedes $U_{\mathrm{b}}$ bei einer anderen Zeit.

Die Kurve wurde dann als „Einhüllende“ unter Auslassung der falschen Nebenpeaks gezeichnet. Der Anschluß an den eigentlichen Nebenpeak bei 1,7 $\mu$ sec (Mitte der Flugzeit) ist gut. Der gesamte Kurvenverlauf ist deutlich stärker gekrümmt als bei Heptan oder Butan, die Intensität ist rund zwei Größenordnungen kleiner.

Nachdem der falsche Nebenpeak bereis die Existenz der Reaktion $\mathrm{CD}_{3} \mathrm{H}^{+} \rightarrow \mathrm{CD}_{3}{ }^{+}+\mathrm{H}$ angezeigt hatte, wurde eine systematische Suche nach Metastabilen in den fünf Gasen $\mathrm{CH}_{4}, \mathrm{CH}_{3} \mathrm{D}, \mathrm{CH}_{2} \mathrm{D}_{2}$, $\mathrm{CHD}_{3}$ und $\mathrm{CD}_{4}$ unternommen ${ }^{28}$. Für Methan gibt es schon mehrere solcher Untersuchungen, die alle ein negatives Ergebnis hatten ${ }^{25,} 27,29$.

Bei der hier beschriebenen Messung wurden jeweils nur die Nebenpeaks beobachtet. Die Quadrupollinse war ausgeschaltet. Die Elektronenenergie im Molekularstrahl betrug etwa $36 \mathrm{eV}$.

Messungen der Druckabhängigkeit der Nebenpeaks zeigten klar, daß in allen fünf Gasen echte unimolekulare verzögerte Zerfälle vorkommen. Der Prozeß 1 wurde kürzlich auch von J. H. Futrell gefunden (private Mitteilung). Wegen des vermutlichen Grundes für den Widerspruch dieser Messungen mit ${ }^{27}$ siehe ${ }^{28}$.

\begin{tabular}{|c|c|c|c|c|}
\hline $\begin{array}{c}1 \\
\mathrm{Nr} .\end{array}$ & $\begin{array}{c}2 \\
\text { Gas }\end{array}$ & 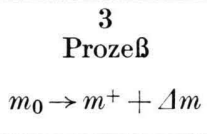 & $\begin{array}{c}4 \\
I_{\text {rel }}\end{array}$ & $\begin{array}{c}5 \\
\text { Int. nor- } \\
\text { miert auf } \\
I_{\mathrm{p}}=100\end{array}$ \\
\hline 1 & $\mathrm{CH}_{4}$ & $16^{+} \rightarrow 15^{+}+1$ & $2,38 \cdot 10^{-4}$ & 0,018 \\
\hline 2 & $\mathrm{CH}_{3} \mathrm{D}$ & $17^{+} \rightarrow 16^{+}+1$ & $3,62 \cdot 10^{-4}$ & 0,029 \\
\hline 3 & $\mathrm{CH}_{2} \mathrm{D}_{2}$ & $18^{+} \rightarrow 17^{+}+1$ & $5,8 \cdot 10^{-4}$ & 0,031 \\
\hline 4 & $\mathrm{CH}_{2} \mathrm{D}_{2}$ & $17^{+} \rightarrow 16^{+}+1$ & $6,3 \cdot 10^{-5}$ & 0,0017 \\
\hline 5 & $\mathrm{CHD}_{3}$ & $19^{+} \rightarrow 18^{+}+1$ & $1,08 \cdot 10^{-3}$ & 0,04 \\
\hline 6 & $\mathrm{CHD}_{3}$ & $17^{+} \rightarrow 16^{+}+1$ & $1,46 \cdot 10^{-4}$ & 0,0006 \\
\hline 7 & $\mathrm{CD}_{4}$ & $20^{+} \rightarrow 18^{+}+2$ & $3,75 \cdot 10^{-4}$ & 0,029 \\
\hline
\end{tabular}

Tab. 3. Intensitäten der verzögerten Dissoziationen in Methan und den deuterierten Methanen.

Tab. 3 stellt die Ergebnisse zusammen. Die auffallendsten Ergebnisse dieser Messungen sind die folgenden:

28 Ch. Ottinger, Z. Naturforschg. 20 a, 1232 [1965].

29 C. E. Melton u. H. M. Rosenstock, J. Chem. Phys. 26, 568 [1957].

30 M. L. Vestal, J. Chem. Phys. 41, 3997 [1964].
1. Die Tatsache, daß es überhaupt in so kleinen Molekülen Metastabile gibt.

2. Die deutlich stärkere Krümmung der Zerfallsfunktion, verglichen mit Butan und Heptan.

3. Das völlige Fehlen von verzögerten D-Abspaltungen, sobald das Molekül H enthält.

4. Der charakteristische Gang der Intensität der verzögerten Dissoziationen in der Reihe

$$
\mathrm{CH}_{4}-\mathrm{CH}_{3} \mathrm{D}-\mathrm{CH}_{2} \mathrm{D}_{2}-\mathrm{CHD}_{3}-\mathrm{CD}_{4} \text {. }
$$

Diese Beobachtungen sollen jetzt qualitativ erklärt werden.

Vestal $^{30}$ hat die Quasi-Äquilibrium-Theorie auf diese Zerfälle angewendet und für die Reaktion $\mathrm{CD}_{4}{ }^{+} \rightarrow \mathrm{CD}_{3}{ }^{+}+\mathrm{D}$ die an der Schwelle zu erwartende minimale Zerfallskonstante $k_{\min }=1 /\left(h \varrho\left(E_{0}\right)\right)$ berechnet. $\varrho\left(E_{0}\right)$ ist dabei die Dichte der Schwingungsniveaus des mit einer Energie $E_{0}$ angeregten $\mathrm{CD}_{4}{ }^{+}$. Sie wurde nach der Näherungsformel von Vestal, Wahrhaftig und Johnston ${ }^{31}$ berechnet. Er erhielt unter der Annahme, daß die Schwingungsfrequenzen für das Ion dieselben sind wie für das $\mathrm{CD}_{4}$-Molekül: $k_{\min }=4,4 \cdot 10^{9} \mathrm{sec}^{-1}$. Da die Methode von Vestal u. a. ${ }^{31}$ bisher noch wenig geprüft worden ist, wurde hier zur Bestimmung von $\varrho\left(E_{0}\right)$ auch die Methode von Whitten-Rabinovitch ${ }^{32}$ angewendet, mit dem Ergebnis $k_{\min }=6,8 \cdot 10^{9} \mathrm{sec}^{-1}$.

Wenn $k_{\min }$ in der Größenordnung $10^{9} \mathrm{sec}^{-1}$ liegt, sind aber Zerfälle nach einigen $\mu$ sec unbeobachtbar. Will man also das Auftreten von Metastabilen überhaupt mittels der Quasi-Äquilibrium-Theorie erklären, so muß man die Zustandsdichte $\varrho$ um mehrere Größenordnungen größer annehmen. Vestal fand, $\mathrm{da}$ ß eine Erniedrigung aller Schwingungsfrequenzen in $\mathrm{CD}_{4}{ }^{+}$gegenüber $\mathrm{CD}_{4}$ auf ein Drittel $k_{\text {min }}$ um etwa einen Faktor 1000 erniedrigte. Eine so drastische Frequenzänderung tritt aber sicher nicht ein. $\mathrm{Ob} \mathrm{An}$ harmonizität, die Vestal als Ausweg vorschlägt, zur Erhöhung von $\varrho$ ausreicht, ist zweifelhaft. Statt dessen soll hier der Einfluß der Rotation des Molekülions betrachtet werden. Sobald nämlich eine Kopplung der Schwingung mit der Rotation auf dem Wege über die Veränderung des Trägheitsmoments bei der Schwingung vorkommt, kann auch Energie in die Rotationsfreiheitsgrade fließen. Dadurch wird also $\varrho(E)$ sicher erhöht.

31 M. L. Vestal, A. L.Wahrhaftig u. W. H. Johnston, J. Chem. Phys. 37, 1276 [1962].

32 G. Z. Whitten u. B.S. Rabinovitch, J. Chem. Phys. 38, 2466 [1963]. 
Für den vorliegenden Fall der Methane gibt es nun einen direkten experimentellen Beweis für die Kopplung von Rotation und Schwingung. Die Nebenpeaks waren nämlich in diesen Fällen besonders schmal, im Mittel nur 1,27-mal breiter als die Strahlpeaks. Eine genaue Bestimmung der Zerfallsenergie nach (4) war daher nicht möglich; größenordnungsweise erhält man jedoch das überraschend kleine $E_{\mathrm{z}}=0,005 \mathrm{eV}$. Man sollte als untere Grenze aller gemessenen $E_{z}$ etwa thermische Energie erwarten. Zwar ist die hier verwendete Methode zur Messung von $E_{z}$ von der thermischen Translationsenergie unabhängig, da diese durch den Zerfall im Fluge nicht "verstärkt" wird. Statt dessen findet man aber die thermische Rotationsenergie in den Fragmenten verstärkt wieder, sie ist von einer „echten“ Zerfallsenergie $E_{\mathrm{z}}$ eines nicht rotierenden Ions nicht zu unterscheiden. Die Tatsache, daß hier $E_{\mathrm{z}}$ wesentlich unterthermisch gefunden wird, kann nun nur so gedeutet werden, daß beim Übergang zum aktivierten Komplex das Trägheitsmoment $\Theta$ sich so vergrößert, daß infolge der Erhaltung des Drehimpulses $\hbar V j(j+1)$ (vorausgesetzt, daß das H-Atom ,zentral" vom C-Atom wegfliegt) die Rotationsenergie $\hbar^{2} j(j+1) / 2 \Theta$ stark abnimmt. Die so freigesetzte Energie wird dazu benutzt, einen Teil der Aktivierungsenergie aufzubringen. Dies bedeutet aber gerade eine Kopplung zwischen der Rotation und der Bewegung in der Reaktionskoordinate. Eine Kopplung mit den Normalschwingungen vor dem Übergang in den aktivierten Komplex ist dann ebenfalls zu erwarten.

Die Berücksichtigung der Rotation ergibt nach Whitten und Rabinovitch ${ }^{33}$ im wesentlichen, daß die Zahl der Schwingungs-Rotations-Niveaus bis zu einer Energie $E$ um den Faktor $Z_{\mathrm{r}}$ größer ist als die Zahl der Schwingungsniveaus. Dabei bedeutet $Z_{\mathrm{r}}$ die Rotationszustandssumme, in $\operatorname{der}(k T)^{r / 2}$ durch $\left(E+a E_{0}\right)^{r / 2}$ ersetzt ist $\left(E_{0}=\right.$ Nullpunktsenergie, für den energieabhängigen Parameter $a$ wird in ${ }^{32}$ eine Tabelle angegeben; $r=$ Zahl der Rotationsfreiheitsgrade). Die Tatsache, daß die volle Energie $E$ in die Zustandssumme eingeht, bedeutet den freien Austausch von Schwingungs- und Rotationsenergie. Näherungsweise wird dann auch die Zustandsdichte gegenüber dem rotationslosen Fall um den Faktor $Z_{\mathrm{r}}$ erhöht.

33 G. Z. Whitten u. B. S. Rabinovitch, J. Chem. Phys. 41, 1883 [1964].
Wie im rotationslosen Fall gilt auch hier $k_{\min }$ $=1 /(h \varrho(E)), k_{\min }$ wird also etwa um den Faktor $Z_{\mathrm{r}}$ erniedrigt. Genau an der Schwelle gibt es nur einen einzigen dissoziationsfähigen Zustand des aktivierten Komplexes (daher die 1 im Zähler). Dieser eine Zustand ist ein rotationsloser. Jegliche in der Rotation enthaltene Energie ist ja ganz oder zum Teil für die Dissoziation, d.h. zur Überwindung des Potentialwalles, verloren; ganz, wenn es sich um Rotation um die Längsachse des dissoziierenden Komplexes handelt, und teilweise für die beiden anderen Rotationsfreiheitsgrade, in denen wegen der Drehimpulserhaltung immer der Bruchteil $\Theta / \Theta^{\ddagger}$ als Rotationsenergie nach erfolgter Dissoziation verbleibt $\left(\Theta, \Theta^{\ddagger}\right.$ sind die Trägheitsmomente von Molekülion und aktiviertem Komplex). Genau an der Schwelle fliegen also die Fragmente ohne Drehimpuls auseinander.

Eine Abschätzung für $Z_{\mathrm{r}}$ von $\mathrm{CH}_{4}$ bei $E=1,68 \mathrm{eV}$ (s. Anm. ${ }^{26}$ ) gibt

$$
Z_{\mathrm{r}}=\left(\frac{2 \Theta E}{\hbar^{2}}\right)^{3 / 2} \approx 150000 .
$$

Für den Fall, daß nur 10\% der Gesamtenergie in die Rotation fließen können, wird $Z_{\mathrm{r}}$ rund 5000 . Jedenfalls sieht man, daß die Berücksichtigung der Rotation $k_{\min }$ um mehrere Größenordnungen erniedrigen kann, was gerade das Auftreten von Metastabilen erklärt.

Zugleich wird aber auch die starke Krümmung der Zerfallsfunktion von $\mathrm{CD}_{4}{ }^{+} \rightarrow \mathrm{CD}_{3}{ }^{+}+\mathrm{D}$ verständlich: Das obige Argument gilt nur für die unmittelbare Umgebung der Schwelle. In dem Maße, in dem mit steigender Energie $E$ im Ion mehr und mehr Rotationszustände des aktivierten Komplexes möglich werden, nimmt $Z_{\mathrm{r}}{ }^{\ddagger}$ schnell zu, und zwar relativ viel schneller als $Z_{\mathrm{r}}$, so daß sich der Ausdruck für $k$ dem nur aus den Schwingungen berechneten, viel größeren $k$ annähert. Also ist nur ein sehr kleiner Energiebereich für die Entstehung der Metastabilen verantwortlich (solange der Energieüberschuß oberhalb der Schwelle noch nicht groß gegen die Rotationsquanten des aktivierten Komplexes ist), was die kleine Intensität erklärt. Die starke Krümmung zeigt direkt anschaulich die schnelle Zunahme des statistischen Gewichts der Rotationszustände des aktivierten Komplexes (und damit die schnelle Zunahme von $k$ ) bei nur geringer Energieerhöhung.

Den starken Isotopieeffekt, der im Fehlen jeglicher verzögerten D-Abspaltung besteht, kann man folgendermaßen verstehen: Die Potentialkurven, aufgetragen längs der Reaktionskoordinaten für H- bzw. 
D-Abspaltung, sind dieselben. Dagegen sind die Nullpunktsenergien der $\mathrm{C}-\mathrm{H}$ - und C-D-Schwingungen verschieden. Nimmt man den Unterschied der höchstfrequenten Normalschwingung in $\mathrm{CH}_{4}$ $\left(3022 \mathrm{~cm}^{-1}\right)$ und $\mathrm{CD}_{4}\left(2258 \mathrm{~cm}^{-1}\right)$ als Anhaltspunkt, so sind ca. $400 \mathrm{~cm}^{-1}$ Unterschied der Nullpunktsenergien zu erwarten. Um soviel liegt also die Aktivierungsenergie für D-Abspaltung höher. Für jede feste Energie im Ion hat diese daher ein kleineres $k$, und wenn $\mathrm{H}$ - und D-Abspaltung konkurrierende Primärprozesse sind (etwa in $\mathrm{CH}_{2} \mathrm{D}_{2}{ }^{+}$), stehen ihre Häufigkeiten im Verhältnis der $k$-Werte. Der beschriebene besonders steile Verlauf von $k(E)$ erklärt dann die völlige Unterdrückung der D-Abspaltung in der Nähe der Schwelle.

Ein ähnlicher Isotopieeffekt wurde übrigens kürzlich auch bei Metastabilen in Propan beobachtet.

Tab. 3, Spalte 4, zeigt, daß deuterierte Methanionen um so häufiger verzögerte $\mathrm{H}$-Abspaltungen erleiden, je weniger $\mathrm{H}$ das Molekül enthält. Dies erklärt sich durch Betrachtung der Symmetriefaktoren $\sigma$, d. h. der Zahl der Möglichkeiten, die Reaktionskoordinate zu wählen. Für die Reaktionen 1, 2, 3 und 5 ist $\sigma=4,3,2$ und 1. Da die Reaktionskonstante $k$ den Faktor $\sigma$ enthält, nimmt mit zunehmender D-Substituierung $k$ ab, was die langlebigen Ionen bevorzugt und daher die relativen Nebenpeakintensitäten anwachsen läßt.

Für die Reaktion 7 ist $\sigma=4$ wie bei der Reaktion 1. Es handelt sich jetzt aber um eine D-Abspaltung, wodurch $k$ bei allen Energien um den Faktor $\sqrt{2}$ erniedrigt wird.

Außer durch die Symmetriefaktoren sowie bei Reaktion 7 auch den Isotopieeffekt wird die Zerfallskonstante $k$ auch durch den sogen. sekundären Isotopieeffekt (SIE) beeinflußt: In den Reaktionen 1, 2, 3, 5 ist zwar das abgespaltene Fragment immer dasselbe (H). Die Gegenwart von zunehmenden Mengen D im Molekül erniedrigt aber dessen Schwingungs- und Rotationsfrequenzen, was zu einer Erhöhung der Zahl der Zustände und damit zu einer Erniedrigung von $k_{\min }$ führt. Es zeigt sich allerdings, daß diese Messungen keinen Einfluß des SIE erkennen lassen. In Abb. 13 ist $I_{\text {rel }}$ für die Reaktionen 1, 2, 3, 5 gegen $1 / \sigma$ aufgetragen. Es ergibt sich angenähert eine Gerade. Die einfachste Erklärung hierfür ist, $\operatorname{da} \beta I_{\text {rel }}$ proportional $\mathrm{zu} 1 / k$ ist und $\mathrm{da} \beta$ die Unterschiede der $k$ für die Reaktionen 1, 2, 3, 5 durch die Berücksichtigung von $\sigma$ bereits voll erklärt sind. Es ist aber aus diesen vier Punkten allein noch nicht zu ersehen, ob nicht vielleicht die Annahme $I_{\text {rel }} \sim 1 / k$ falsch und dafür ein von 1 nach 5 zunehmender SIE anzunehmen ist. Eine Entscheidung ergibt die Reaktion 7. Für sie sollte der SIE ähnlich groß wie für Reaktion 5 sein. Man sieht aber aus Abb. 13, daß der Punkt 7 sich dem linearen

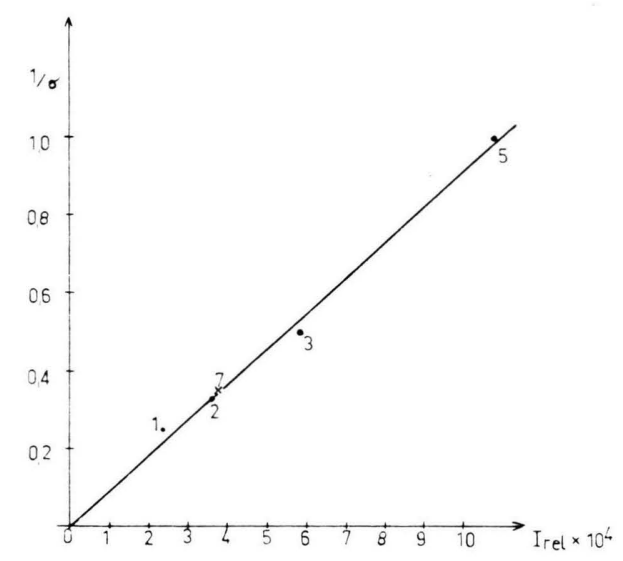

Abb. 13. Korrelation zwischen dem Symmetriefaktor und den beobachteten Metastabilen-Intensitäten in Methan und den deuterierten Methanen.

Zusammenhang bereits einfügt, wenn man als Ordinate in diesem Fall $\sqrt{2} / \sigma=\sqrt{2} / 4$ aufrägt, also nur den primären, nicht den sekundären Isotopieeffekt berücksichtigt. Damit ist gezeigt, daß die vorliegenden Messungen allein durch Betrachtung des Symmetriefaktors und des primären Isotopieeffekts erklärt werden können. Zugleich ist die empirische Regel $I_{\text {rel }} \sim 1 / k$ bestätigt. - Noch in einer anderen Hinsicht nimmt die Reaktion 7 eine Sonderstellung ein: Für sie ist die Aktivierungsenergie ein wenig höher, die Zustandsdichte an der Schwelle also ein wenig größer als bei Reaktion 1. Dieser Effekt würde also in derselben Richtung wirken wie ein SIE und ist ebenso wie dieser zu vernachlässigen.

Der Einfluß des Symmetriefaktors läßt sich übrigens auch bei den beiden Sekundärreaktionen 4 und 6 (beide $\mathrm{CHD}_{2}{ }^{+} \rightarrow \mathrm{CD}_{2}{ }^{+}+\mathrm{H}$ ) beobachten: Die entsprechenden Reaktionen $\mathrm{CH}_{2} \mathrm{D}^{+} \rightarrow \mathrm{CHD}^{+}+\mathrm{H}$ oder gar $\mathrm{CH}_{3}{ }^{+} \rightarrow \mathrm{CH}_{2}{ }^{+}+\mathrm{H}$ konnten gar nicht mehr gefunden werden, weil das Ion zuviel $\mathrm{H}$ enthält.

\section{Benzol}

Bei Benzol wurden die Zerfallskurven der drei Prozesse

und

$$
\begin{aligned}
& \mathrm{C}_{6} \mathrm{H}_{6}{ }^{+} \rightarrow \mathrm{C}_{4} \mathrm{H}_{4}{ }^{+}+\mathrm{C}_{2} \mathrm{H}_{2}, \\
& \mathrm{C}_{6} \mathrm{H}_{5}{ }^{+} \rightarrow \mathrm{C}_{4} \mathrm{H}_{3}{ }^{+}+\mathrm{C}_{2} \mathrm{H}_{2} \\
& \mathrm{C}_{6} \mathrm{H}_{6}{ }^{+} \rightarrow \mathrm{C}_{3} \mathrm{H}_{3}{ }^{+}+\mathrm{C}_{3} \mathrm{H}_{3}
\end{aligned}
$$




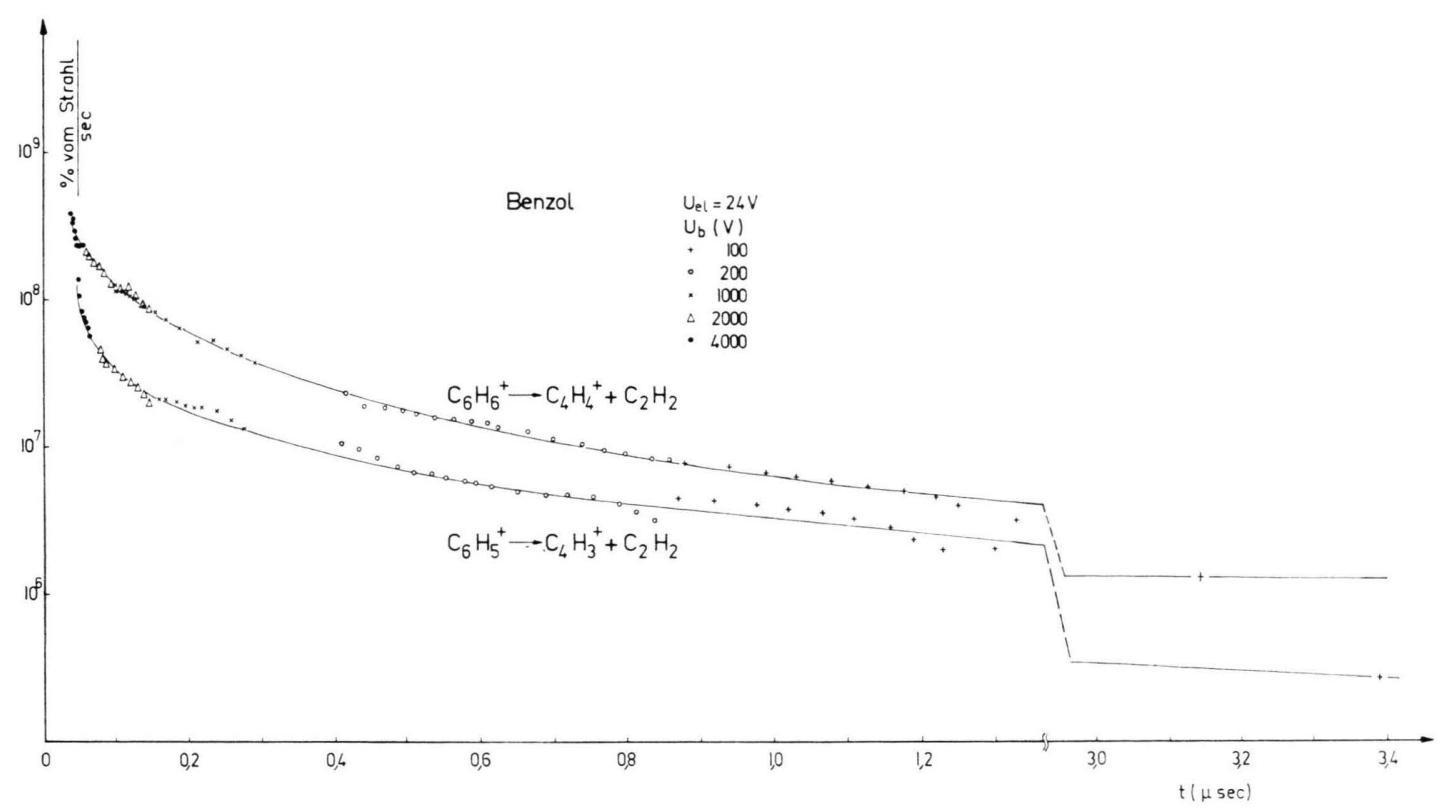

Abb. 14. Zerfallskurven für Benzol.

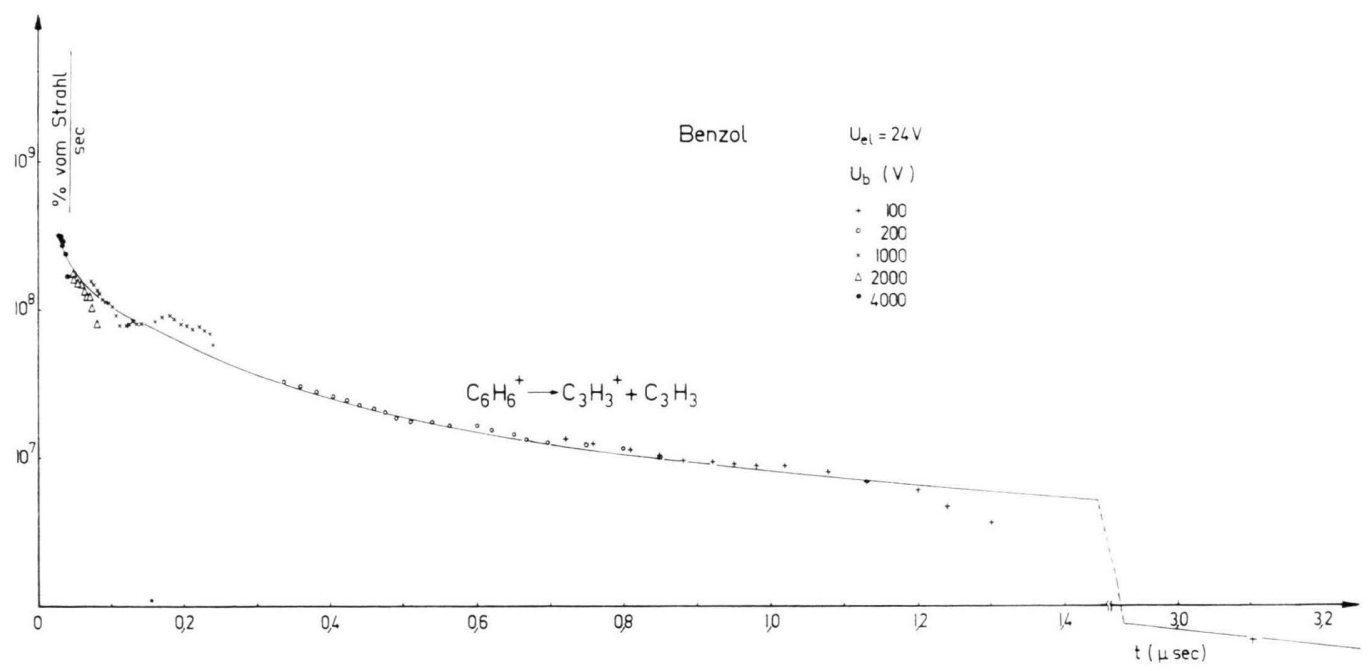

Abb. 15. Zerfallskurve für Benzol.

gemessen (s. Abb. 14 und 15). Der zweite von diesen wurde durch die Existenz der Reaktion $\mathrm{C}_{4} \mathrm{H}_{4}{ }^{+} \rightarrow$ $\mathrm{C}_{4} \mathrm{H}_{3}{ }^{+}+\mathrm{H}$ kompliziert, die einen Nebenpeak dicht am Strahlpeak der Masse 51 erzeugte. Die kleinsten $\Delta U$ jedes $U_{\mathrm{b}}$-Bereichs konnten daher nicht mit in die Auswertung einbezogen werden. Bei dem dritten Prozeß (Masse 39) bestand zusätzlich zu der geringen Intensität eine weitere Komplikation darin, daß auf Masse 39 der extrem große Kaliumpeak von der Anode erscheint, der vom Strahlpeak nicht völlig zu trennen ist. Wegen der Normierung auf die Strahlpeakfläche mußte er durch Abstellen des Gasstrahls für jedes $U_{\mathrm{b}}$ getrennt gemessen und subtrahiert werden.

Benzol ist durch seine eigenartige Elektronenstruktur eine besonders interessante Substanz. Auffallend sind z. B. die hohen Aktivierungsenergien der Primärzerfälle

$$
\begin{aligned}
& 78^{+} \rightarrow 77^{+}(5,1 \mathrm{eV}), \quad 78^{+} \rightarrow 76^{+}(5,4 \mathrm{eV}), \\
& 78^{+} \rightarrow 52^{+}(6,4 \mathrm{eV}) \quad \text { und } 78^{+} \rightarrow 39^{+}(6,9 \mathrm{eV}),
\end{aligned}
$$


die alle auch verzögert vorkommen ${ }^{34}$. (Bei Heptan setzen alle Primärzerfälle innerhalb von $0,8 \mathrm{eV}$ oberhalb I.P. ein.) Alle diese verzögerten Zerfälle erfolgen offenbar aus angeregten Elektronenzuständen heraus und stehen daher u. U. nicht in direkter Konkurrenz miteinander. Interessant ist nun, daß auch hier die Zerfallskurven deutlich gekrümmt sind. Das bedeutet, daß es wahrscheinlich möglich ist, die Schwingungsterme über diesen höheren Elektronentermen für sich nach der statistischen Theorie zu behandeln und Funktionen $k(E)$ zu konstruieren.

Benzol galt bisher wegen der Existenz von fünf verzögerten Zerfällen des Mutterions als einmaliges Beispiel. Die Heptanmessung mit sechs solchen Zerfällen zeigt, daß die Ringstruktur für eine solche Erscheinung nicht nötig ist.

Daten über viele weitere verzögerte Zerfälle des Benzolions finden sich in ${ }^{35}$ und ${ }^{36}$.

\section{Kohlendioxyd}

Einen besonders interessanten verzögerten Zerfall gibt es im Kohlendioxyd ${ }^{37,38}$ :

$$
\mathrm{CO}_{2}{ }^{++} \rightarrow \mathrm{CO}^{+}+\mathrm{O}^{+} \text {. }
$$

$\mathrm{CO}_{2}$ ist eines der kleinsten Moleküle, in denen bisher Metastabile gefunden wurden (nach ${ }^{38}$ gibt es sogar im $\mathrm{CO}$ den verzögerten Prozeß $\mathrm{CO}^{++} \rightarrow \mathrm{C}^{+}+\mathrm{O}^{+}$). Der verzögerte Zerfall erfolgt mit sehr großer Zerfallsenergie $E_{\mathrm{z}}$ von rund $5 \mathrm{eV}{ }^{38}$. Diese Tatsache, darunter vor allem die Existenz des analogen Prozesses in $\mathrm{CO}$, machen es wahrscheinlich, $\mathrm{da} ß$ der Zerfall durch einen Übergang aus einem elektronisch metastabilen Zustand bewirkt wird. Daher erschien es besonders interessant, die Zerfallskurve dieses Prozesses zu messen.

Verzögert gebildete $\mathrm{CO}^{+}$-Ionen aus $\mathrm{CO}_{2}^{++}$können eindeutig daran erkannt werden, daß für sie $\Delta U$ negativ wird. Sie haben nämlich infolge ihrer Herkunft aus einem doppelt geladenen Ion und wegen des Massenverhältnisses $m_{0^{+}} / m_{\mathrm{CO}^{+}}<1$ mehr Energie bei verzögerter Bildung als bei unmittelbarer, man muß also das Ionenquellenpotential erniedrigen, um den Schwanz und Nebenpeak zu messen. Quantitativ erhält man

$$
\Delta U=(\Delta m-m) e\left(\frac{|\mathfrak{E}| t}{m_{0}}\right)^{2} .
$$

34 H. M. Rosenstock u. M. Krauss, in: Advances in Mass Spectrometry, Editor: R. M. Elliotr, Pergamon Press, Oxford 1963.

35 Ch. Ottinger, Z. Naturforschg. 20 a, 1229 [1965]. $m$ ist dabei die Masse des für die Messung benutzten Ions und $\Delta m$ die des anderen. Daher gehören zu gleichen Zeiten für $\mathrm{CO}^{+}$und $\mathrm{O}^{+} \Delta U$-Werte, die symmetrisch zum Strahl liegen.

Eine Schwierigkeit bestand in der großen Zerfallsenergie $E_{z}$. Diese führt zu einer extremen Verbreiterung des Nebenpeaks, die den ausnutzbaren Bereich des Schwanzes stark beschneidet. Für den Zerfall doppelt geladener Ionen erhält man statt (4)

$$
E_{\mathrm{z}}=\frac{\Gamma^{2} m^{2}}{4 m_{0} \Delta m e U_{\mathrm{c}}} .
$$

Bei einem so großen und dazu scharf definierten $E_{\mathrm{z}}$ wie hier sieht man nun tatsächlich je einen Peak für die Vorwärts- und die Rückwärtsionen, weil gegen die seitlich zur Flugrichtung abdissoziierten Ionen stark diskriminiert wird.

Abb. 16 a zeigt diese beiden Anteile des Nebenpeaks, außerdem rechts den Strahlpeak. Darüber in Abb. 16 b ist ein $\mathrm{CO}^{+}$-Nebenpeak gezeigt, der durch Gaseinlaß in die gesamte Apparatur gewonnen wurde (das Ionisationsgebiet wurde hier dadurch begrenzt, daß $U_{\text {el }}$ genügend klein gewählt wurde; Abb. $16 \mathrm{~b}$ zeigt rechts das so erhaltene Äquivalent des Strahlpeaks). Die Strahlpeaks wurden auf Masse 22 $\left(\mathrm{CO}_{2}{ }^{++}\right)$gemessen, die Nebenpeaks auf $28\left(\mathrm{CO}^{+}\right)$. Bei Abb. 16 b war der Druck $4 \cdot 10^{-5}$ Torr, bei Abb. 16 a war der Einlaßdruck vor den Spalten aus Intensitätsgründen extrem hoch, $p_{0}=1$ Torr, und der Druck in der Apparatur 2,2 $10^{-7}$ Torr.

Die Elektronenenergie betrug bei Abb. 16 a $100 \mathrm{eV}$, bei $16 \mathrm{~b}$ nur $65 \mathrm{eV}$ zur Erzielung eines schmaleren Ionisationsgebietes.

Die beiden Messungen unterscheiden sich vor allem durch das Auftreten des mittleren Maximums des Nebenpeaks bei hohem Druck. Dieses besteht also aus stoßinduziert erzeugten $\mathrm{CO}^{+}$-Ionen; ihre Anfangsenergie ist viel kleiner als die der $\mathrm{CO}^{+}$-Ionen in den seitlichen Peaks, da sie ja einem ganz anderen Prozeß (vermutlich mit vorangegangenem Ladungsaustausch des $\mathrm{CO}_{2}{ }^{++},{ }^{38}$ ) entstammen. Die Kurve Abb. 16 b ist übrigens praktisch identisch mit der Peakform, die Fuchs und TAubert ${ }^{38}$ auf der Massenskala für den „metastabilen“ $\mathrm{CO}^{+}$.Peak fanden.

36 K. R. Jennings, J. Chem. Phys. 43, 4176 [1965].

37 A. S. Newton u. A. F. Sciamanna, J. Chem. Phys. 40, 718 [1964].

38 R. Fuchs u. R. Taubert, Z. Naturforschg. 20 a, 823 [1965]. 


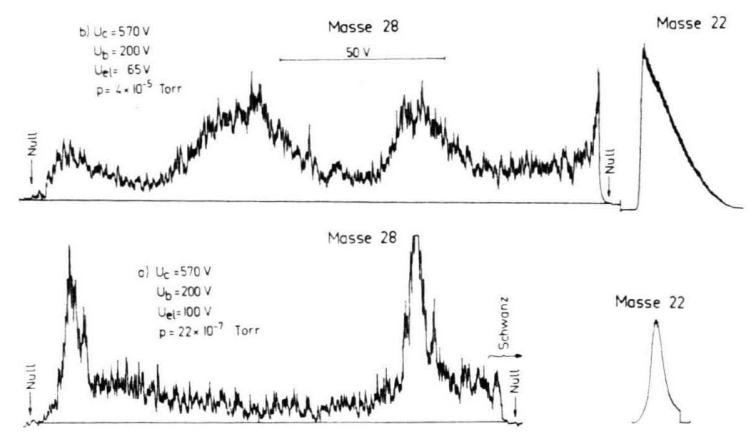

Abb. 16. Neben- und Strahlpeaks für $\mathrm{CO}^{+}$aus $\mathrm{CO}_{2}{ }^{++}$; a) echt metastabil, der Nebenpeak ist durch Anfangsenergie weit aufgespalten; b) Beimischung durch stoßinduzierte Zerfälle, die einen nicht aufgespaltenen Beitrag zum Nebenpeak liefern.

Aus dem Abstand $\Gamma=104 \mathrm{~V}$ der beiden Teilpeaks des Nebenpeaks in Abb. 16 a ergibt sich nach (6)

$$
E_{\mathrm{z}}=5,28 \mathrm{eV} \pm 3 \% \text {, }
$$

in guter Übereinstimmung mit dem Wert $5,11 \mathrm{eV}$ aus ${ }^{38}$.

Nach diesen vorbereitenden Messungen, die den zur Schwanzmessung nutzbaren Potentialbereich anzeigten, wurde dann die Zerfallskurve gemessen. Bei jeder Feldstärke war nur ein sehr kleiner Bereich von $\Delta U$ brauchbar, der z. Tl. erst nach der Auswertung erkennbar wurde. Es sind daher in Abb. 17 auch Punkte eingetragen, die offensichtlich nicht hätten gemessen werden sollen.

Rechts in Abb. 17 ist die Nebenpeakintensität eingetragen. Sie ist etwas zu groß, was vielleicht daran liegt, daß die Diskriminierung gegen Ionen mit groBer Winkeldivergenz für Ionen aus dem Laufraum weniger stark ist.

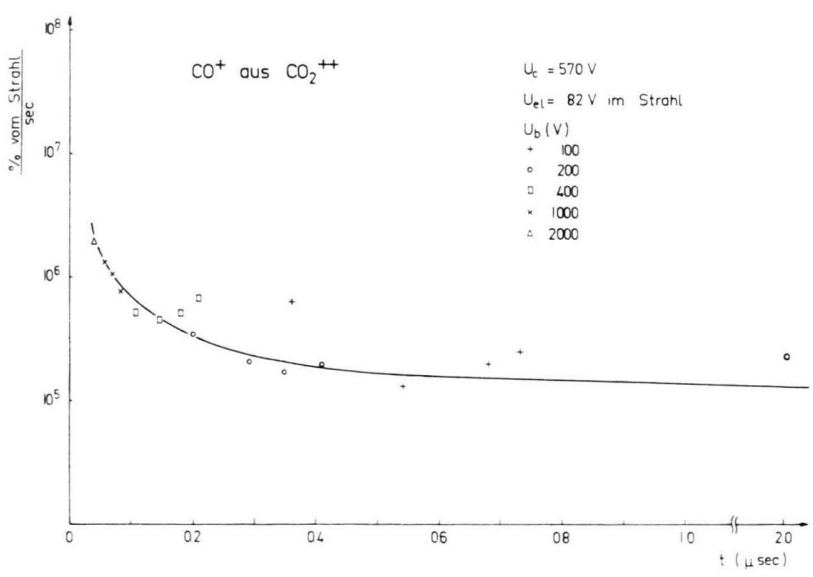

Abb. 17. Zerfallskurve für $\mathrm{CO}_{2}{ }^{++}$.

39 C. E. Melton, M. M. Bretscher u. R. Baldock, J. Chem. Phys. 26, 1302 [1957].
Die in jedem Fall große Diskriminierung läßt es hier nicht $\mathrm{zu}$, die Ordinatenskala mit der anderer Messungen zu vergleichen.

Eine prinzipielle Schwierigkeit besteht noch darin, daß wegen der großen Zerfallsenergie bei jedem $\Delta U$ zwei Zerfallszeitpunkte zum Ionenstrom beitragen, die hier recht verschieden sind. Zum Beispiel setzt sich die bei $t=0,05 \mu$ sec in Abb. 17 eingetragene Intensität in Wirklichkeit aus Zerfällen bei 0,02 und $0,1 \mu$ sec zusammen; ebenso ist der Punkt bei 0,3 $\mu$ sec ein Mittel aus 0,15 und $0,55 \mu$ sec.

Wenn die Zerfallsrate nur durch ein elektronisches Übergangsverbot bestimmt wäre, sollte man eine rein exponentielle Zerfallskurve finden. Trotz der großen Ungenauigkeiten ist aber nach Abb. 17 klar, daß auch hier eine Krümmung (im logarithmischen Maßstab) besteht. Möglicherweise zeigt sie einen Einfluß der Rotation des Molekülions auf die Übergangswahrscheinlichkeit an.

$$
\mathrm{C}_{2} \mathrm{H}_{2}, \mathrm{H}_{2}, \mathrm{O}_{2}, \mathrm{~N}_{2}
$$

Auch für die Gase Azetylen, Wasserstoff, Sauerstoff und Stickstoff wurde nach Nebenpeaks von den Reaktionen $\mathrm{C}_{2} \mathrm{H}^{+} \rightarrow \mathrm{C}_{2}^{+}+\mathrm{H}^{39}, \mathrm{H}_{2}^{+} \rightarrow \mathrm{H}^{+}+\mathrm{H}$, $\mathrm{O}_{2}{ }^{+} \rightarrow \mathrm{O}^{+}+\mathrm{O}$ und $\mathrm{N}_{2}^{+} \rightarrow \mathrm{N}^{+}+\mathrm{N}$ gesucht. Innerhalb einer Ausschließungsgrenze von 0,0015 (bezogen auf den stärksten Peak im Massenspektrum =100) wurden die Reaktionen nicht gefunden.

\section{Messung von Zerfallsenergien bei verzögerten Dissoziationen}

Wie erwähnt, kann man aus den Nebenpeakbreiten die bei verzögerten Dissoziationen freigesetzte gesamte kinetische Energie $E_{\mathrm{z}}$ bestimmen. Dieses Verfahren hat gegenüber den Methoden zur Bestimmung von Anfangsenergien normaler Fragmentionen, etwa der Ablenkmethode ${ }^{42}$, zwei wichtige Vorteile: Man kann hier auch unterthermische Anfangsenergien messen, und außerdem ist der zugehörige Prozeß genau bekannt. Andererseits ist das Verfahren naturgemäß beschränkt auf Zerfälle, die verzögert vorkommen. - Vorläufige Ergebnisse wurden bereits mitgeteilt ${ }^{35,40}$. Einige der früheren Werte sind aber noch durch einen Fehler in Formel (2) in ${ }^{40}$ verfälscht, außerdem wurden seitdem noch weitere Prozesse gemessen und die Genauigkeit erhöht. Tab. 4 zeigt die neuen Ergebnisse.

${ }^{40}$ Сн. Ottinger, Phys. Lett. 17, 269 [1965]. 


\begin{tabular}{|c|c|c|}
\hline \multicolumn{3}{|c|}{ Butan } \\
\hline Nr. & Proze $\beta$ & $E_{\mathrm{z}}(\mathrm{eV})$ \\
\hline $\begin{array}{l}1 \\
2 \\
3 \\
4 \\
5 \\
6 \\
7\end{array}$ & $\begin{array}{l}58^{+} \rightarrow 43^{+}+15 \\
58^{+} \rightarrow 42^{+}+16 \\
57^{+} \rightarrow 41^{+}+16 \\
43^{+} \rightarrow 41^{+}+2 \\
29^{+} \rightarrow 27^{+}+2 \\
42^{+} \rightarrow 41^{+}+1 \\
55^{+} \rightarrow 29^{+}+26\end{array}$ & $\begin{array}{l}0,020 \pm 0,005 \\
0,031 \pm 0,01 \\
0,30 \pm 0,13 \\
0,40 \pm 0,05 \\
0,033 \pm 0,009 \\
0,02 \pm 0,02 \\
0,025 \pm 0,007\end{array}$ \\
\hline \multicolumn{3}{|c|}{ Benzol } \\
\hline $\begin{array}{l}1 \mathrm{a} \\
1 \mathrm{~b} \\
1 \mathrm{c} \\
1 \mathrm{~d} \\
1 \mathrm{e} \\
1 \mathrm{f} \\
2 \mathrm{a} \\
2 \mathrm{~b} \\
3 \mathrm{a} \\
3 \mathrm{~b} \\
3 \mathrm{c} \\
4\end{array}$ & 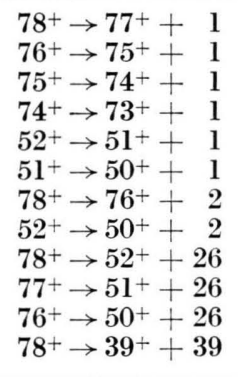 & $\begin{array}{l}0,072 \pm 0,02 \\
0,096 \pm 0,04 \\
0,084 \pm 0,03 \\
0,044 \pm 0,044 \\
0,096 \pm 0,03 \\
0,066 \pm 0,024 \\
0,25 \pm 0,04 \\
0,32 \pm 0,11 \\
0,031 \pm 0,005 \\
0,023 \pm 0,005 \\
0,027 \pm 0,005 \\
0,038 \pm 0,009\end{array}$ \\
\hline \multicolumn{3}{|c|}{ Heptan } \\
\hline $\begin{array}{r}1 \\
2 \\
3 \\
4 \\
5 \\
6 \\
7 \\
8 \\
9 \\
10 \\
11 \\
12 \\
13 \\
14 \\
15 \\
16 \\
17 \\
18 \\
19 \\
20 \\
21 \\
22 \\
\end{array}$ & $\begin{aligned} 55^{+} \rightarrow 53^{+}+2 \\
43^{+} \rightarrow 41^{+}+2 \\
41^{+} \rightarrow 39^{+}+2 \\
29^{+} \rightarrow 27^{+}+2 \\
28^{+} \rightarrow 26^{+}+2 \\
100^{+} \rightarrow 85^{+}+15 \\
70^{+} \rightarrow 55^{+}+15 \\
58^{+} \rightarrow 42^{+}+16 \\
57^{+} \rightarrow 41^{+}+16 \\
55^{+} \rightarrow 29^{+}+26 \\
53^{+} \rightarrow 27^{+}+26 \\
85^{+} \rightarrow 57^{+}+28 \\
84^{+} \rightarrow 56^{+}+28 \\
71^{+} \rightarrow 43^{+}+28 \\
70^{+} \rightarrow 42^{+}+28 \\
57^{+} \rightarrow 29^{+}+28 \\
100^{+} \rightarrow 71^{+}+29 \\
100^{+} \rightarrow 70^{+}+30 \\
85^{+} \rightarrow 43^{+}+42 \\
100^{+} \rightarrow 57^{+}+43 \\
100^{+} \rightarrow 56^{+}+44 \\
100^{+} \rightarrow 42^{+}+58 \\
\end{aligned}$ & $\begin{array}{l}0,41 \pm 0,05 \\
0,43 \pm 0,05 \\
0,35 \pm 0,08 \\
0,022 \pm 0,004 \\
0,038 \pm 0,011 \\
0,050 \pm 0,010 \\
0,020 \pm 0,002 \\
0,182 \pm 0,018 \\
0,16 \pm 0,02 \\
0,022 \pm 0,04 \\
0,028 \pm 0,008 \\
0,015 \pm 0,004 \\
0,062 \pm 0,031 \\
0,020 \pm 0,002 \\
0,027 \pm 0,003 \\
0,049 \pm 0,016 \\
0,022 \pm 0,003 \\
0,039 \pm 0,004 \\
0,021 \pm 0,004 \\
0,050 \pm 0,005 \\
0,027 \pm 0,002 \\
0,026 \pm 0,004 \\
\end{array}$ \\
\hline
\end{tabular}

Tab. 4. Zerfallsenergien metastabiler Ionen.

Aus dem Vergleich von Butan mit Heptan kann man ablesen, da $\beta$ derselbe Proze $\beta$ in beiden Gasen auch etwa das gleiche $E_{\mathrm{z}}$ liefert: Nr. 3 - Nr. 9; Nr. 4 - Nr. 2 ; Nr. 5 - Nr. 4; Nr. 7 - Nr. 10; auch der zu Nr. 16 im Heptan analoge Butanprozeß schien ein ähnliches $E_{\mathrm{z}}$ zu haben, obwohl die Intensität zur Messung nicht ausreichte. Eine Ausnahme bildet das Paar 2-8. Dies erklärt sich aber aus der

41 C. E. KLors, J. Chem. Phys. 41, 117 [1964]. oben gegebenen Deutung, da $\beta$ es sich beim Heptan in Wirklichkeit um den Prozeß 9, jedoch mit einem ${ }^{13} \mathrm{C}$-Atom handelt. Nr. 8 und 9 beim Heptan haben ja auch innerhalb der Fehlergrenzen dasselbe $E_{\mathrm{z}}$.

Besonders auffallend ist, daß bei manchen $\mathrm{H}_{2}$ - $\mathrm{Ab}$ spaltungen $E_{\mathrm{z}}$ groß (Nr. 1, 2, 3 in Heptan und 4 in Butan) und bei anderen klein ist (Nr. 4, 5 in Heptan und 5 in Butan). Diese Beobachtung, die zweifelsfrei reproduzierbar war, läßt sich wahrscheinlich durch einen verschiedenen Mechanismus der $\mathrm{H}_{2}$-Abspaltung erklären. Eine direkte Nachprüfung des Mechanismus mit deuterierten Verbindungen ist aber infolge "Scrambling“ von $\mathrm{H}$ und $\mathrm{D}$ im Ion leider nicht möglich (J. Futrell, private Mitteilung). Besonders schön lassen sich die Energien beim Benzol klassifizieren: Sie zerfallen in vier Gruppen, für jeden der vier vorkommenden Reaktionstypen eine.

Zur Erklärung der Anfangsenergie normaler Fragmentionen im Massenspektrum haben KLots ${ }^{41}$ und TAubert ${ }^{42}$ aus der statistischen Theorie einen $\mathrm{Zu}$ sammenhang zwischen der mittleren Translationsenergie in der Reaktionskoordinaten und der Energie $E$ im Molekül abgeleitet. Zur Vereinfachung wird dann für jede Reaktion eine mittlere Energie $E$ eingesetzt. Dieses Verfahren ließe sich daher im Prinzip sofort auf die Zerfallsenergien bei verzögerten Dissoziationen übertragen, indem man die (natürlich viel kleinere) mittlere Energie der verzögert zerfallenden Ionen einsetzt.

Mit den Meßergebnissen von Taubert ${ }^{42}$ sind nur die Reaktionen 1 und 2 beim Butan vergleichbar, wo er ein mittleres $E_{\mathrm{z}}$ von 0,10 bzw. $0,14 \mathrm{eV}$ findet. Es ist verständlich, daß die Werte der vorliegenden Arbeit kleiner ausfallen, da nur Moleküle mit besonders kleinen Anregungsenergien beitragen, während es andererseits befriedigend ist, da $\beta$ das Verhältnis $E_{\mathrm{z}}(42): E_{\mathrm{z}}(43)$ in beiden Arbeiten nahezu dasselbe ist. Es scheint, daß man die $E_{\mathrm{z}}$ bis vielleicht in die Größenordnung von $0,1 \mathrm{eV}$ nach der statistischen Theorie erklären könnte; wesentlich größere $E_{z}$, wie sie insbesondere bei den $\mathrm{H}_{2}$-Abspaltungen und der Reaktion $\mathrm{C}_{4} \mathrm{H}_{9}{ }^{+} \rightarrow \mathrm{C}_{3} \mathrm{H}_{5}{ }^{+}+\mathrm{CH}_{4}$ gemessen wurden, erfordern aber zur Erklärung einen anderen $\mathrm{Me}$ chanismus, nämlich die Existenz einer Aktivierungsenergie für die umgekehrte Reaktion. Eine solche, die besonders bei der Abspaltung von stabilen Molekülen zu erwarten ist, dürfte auch schon bei kleineren $E_{z}$ eine Rolle spielen, z. B. ist $E_{z}$ für die Reak-

42 R. Taubert, Z. Naturforschg. 19 a, 911 [1964]. 
tionen 17 und 18 im Heptan deutlich außerhalb der Fehlergrenzen verschieden. KLots zeigte, daß ein „loser" aktivierter Komplex größere kinetische Energie ergibt. Dies, bei der sonstigen Ähnlichkeit der beiden Zerfälle, würde aber ein größeres $E_{z}$ für Masse 71 erwarten lassen. $\mathrm{Da}$ es in Wirklichkeit umgekehrt ist, wird auf die bei der Bildung von $\mathrm{C}_{2} \mathrm{H}_{6}$ freigesetzte Energie zurückgeführt. Sie sollte zwar vorwiegend als Schwingungsenergie erscheinen, ein Teil kann aber auch in Translationsenergie umgesetzt werden. Eine Abschätzung ergibt, daß bei der Reaktion 18 (Tab. 4) im Vergleich zu 17 ein Ener- gieüberschuß von ca. $0,5-1,5 \mathrm{eV}$ freigesetzt wird. Tab. 4 zeigt, daß davon nur $0,02 \mathrm{eV}$ als kinetische Energie erscheinen.

Herrn Prof. Dr. O. Osberghaus danke ich herzlich für die Anregung zu dieser Arbeit und für viele wichtige Diskussionen. Zu Dank verpflichtet bin ich auch den Herren Dr. F. Dorer und Dr. J. Berkowitz für fruchtbare Diskussionen, sowie den Herren I. Hertel und C. Martiv für ihre Hilfe bei den Messungen und der Auswertung. Der Deutschen Forschungsgemeinschaft danke ich für die Bereitstellung der Mittel, die diese Arbeit ermöglichten, der Fernseh GmbH. Darmstadt für die großzügige Überlassung der Netze der Ionenquelle.

\title{
Appearance Potentials of Metastable Molecular Ions
}

\author{
I. Hertel and Ch. Ottinger \\ Physikalisches Institut der Universität Freiburg i. Br. \\ (Z. Naturforschg. 22 a, 40-47 [1967] ; received 26. Oktober 1966)
}

\begin{abstract}
Differences between the appearance potentials of fragment ions formed a few $\mu$ secs after the ionization (so-called metastables) and normal fragment ions were measured. It is shown, however, that no immediate meaning regarding the primary fragmentation mechanism can be attached to these quantities. Instead, the experimental ionization curves of metastable and normal fragments were explained by a theoretical model which assumes 1) a linear threshold law, 2) a linear relation between the logarithm of the rate constant and the internal energy in the molecular ion, and 3) a constant density of populated states on the energy scale. In the case of HCN loss from benzonitrile ions, an excellent fit between the experimental and theoretical curves can then be achieved if it is assumed that an increase of $0.65 \mathrm{eV}$ internal energy increases the rate constant by one order of magnitude. This is a surprisingly slow rise of $k(E)$, compared with the few existing calculations in other cases.
\end{abstract}

The description of the decomposition of an excited polyatomic molecular ion in terms of the quasiequilibrium theory predicts a rapid variation of the rate constant $k$ with the internal energy $E$ in the ion (see for example ${ }^{1}$ ). The so-called "metastable" ions (this term is here used for fragments formed a few $\mu$ secs after the initial ionization) differ from the "normal" fragment ions (which are formed in a very short time after the ionization and constitute the ordinary mass spectrum) in that the rate constant for formation of the former is several orders of magnitude smaller than for the latter. A measurement of the relative intensities of metastable and normal fragment ions as a function of the ionizing electron energy, especially near threshold, should therefore give some indication of the variation of $k$ with $E$.

1 M. L. Vestal, J. Chem. Phys. 43, 1356 [1965].

2 O. Osberghaus and Ch. Ottinger, Phys. Lett. 16, 121 [1965].

- Ch. Ortinger, Z. Naturforschg. 22 a, 20 [1967].

\section{Experimental}

Our apparatus has been briefly described previously ${ }^{2}$. The ions are formed in a molecular beam of $50 \mu$ half-width which is crossed by an electron beam from an oxide coated cathode in order to reduce the thermal electron energy spread to about $0.3 \mathrm{eV}$. The ion drawout field of $100 \mathrm{~V} / \mathrm{cm}$ produces a voltage drop of $0.5 \mathrm{eV}$ across the molecular beam, so that the effective energy spread can be approximated by $\sqrt{0.3^{2}+0.5^{2}}=0.6 \mathrm{eV}$. The electron energy was increased linearly with time by means of a motor driven potentiometer at a rate of about $0.25 \mathrm{~V} / \mathrm{min}$. The "normal" fragment ions were singled out by passing the ion beam through an energy selector with $2.4 \mathrm{eV}$ resolution (half-width). Fragment ions formed a certain time after ionization give up part of their kinetic energy to the neutral fragment and have therefore insufficient energy to pass the selector. The "metastable" fragment ions were measured by setting the molecular beam potential to a value $m_{0} / m$ times the energy required by ions to pass the selector ( $m_{0}=$ mass of reactant ion, $m=$ fragment ion mass). At this setting, only ions formed in a $15 \mathrm{~cm}$ long field- 